\title{
The RNA-binding ubiquitin ligase MKRN1 functions in ribosome-associated quality control of poly $(A)$ translation
}

\author{
Andrea Hildebrandt ${ }^{1}$, Mirko Brüggemann², Cornelia Rücklé ${ }^{1,2}$, Susan Boerner ${ }^{2}$, Jan B. Heidelberger ${ }^{1}$, Anke Busch', \\ Heike Hänel ${ }^{1}$, Andrea Voigt ${ }^{1}$, Martin M. Möckel ${ }^{1}$, Stefanie Ebersberger ${ }^{1}$, Anica Scholz ${ }^{3}$, Annabelle Dold ${ }^{1}$, \\ Tobias Schmid ${ }^{3}$, Ingo Ebersberger ${ }^{4,5}$, Jean-Yves Roignant ${ }^{1,6}$, Kathi Zarnack $^{2^{*}}$, Julian König ${ }^{1^{*}}$ (D) and Petra Beli ${ }^{1^{*}}$
}

\begin{abstract}
Background: Cells have evolved quality control mechanisms to ensure protein homeostasis by detecting and degrading aberrant mRNAs and proteins. A common source of aberrant mRNAs is premature polyadenylation, which can result in non-functional protein products. Translating ribosomes that encounter poly(A) sequences are terminally stalled, followed by ribosome recycling and decay of the truncated nascent polypeptide via ribosomeassociated quality control.

Results: Here, we demonstrate that the conserved RNA-binding E3 ubiquitin ligase Makorin Ring Finger Protein 1 (MKRN1) promotes ribosome stalling at poly(A) sequences during ribosome-associated quality control. We show that MKRN1 directly binds to the cytoplasmic poly(A)-binding protein (PABPC1) and associates with polysomes. MKRN1 is positioned upstream of poly(A) tails in mRNAs in a PABPC1-dependent manner. Ubiquitin remnant profiling and in vitro ubiquitylation assays uncover PABPC1 and ribosomal protein RPS10 as direct ubiquitylation substrates of MKRN1.
\end{abstract}

Conclusions: We propose that MKRN1 mediates the recognition of poly(A) tails to prevent the production of erroneous proteins from prematurely polyadenylated transcripts, thereby maintaining proteome integrity.

Keywords: MKRN1, Ubiquitylation, RNA binding, Ribosome-associated quality control, Poly(A), iCLIP, Ubiquitin remnant profiling, Translation

\section{Introduction}

During gene expression, quality control pathways monitor each step to detect aberrant mRNAs and proteins. These mechanisms ensure protein homeostasis and are essential to prevent neurodegenerative diseases [1]. A common source of aberrant mRNAs is premature polyadenylation, often in combination with mis-splicing, which results in truncated non-functional protein products [2]. Therefore, mechanisms are in place that

\footnotetext{
* Correspondence: kathi.zarnack@bmls.de; j.koenig@imb-mainz.de; p.beli@imb-mainz.de

²Buchmann Institute for Molecular Life Sciences (BMLS), Goethe University, Max-von-Laue-Str. 15, 60438 Frankfurt am Main, Germany

${ }^{1}$ Institute of Molecular Biology (IMB), Ackermannweg 4, 55128 Mainz, Germany

Full list of author information is available at the end of the article
}

recognize such homopolymeric adenosine (poly(A)) sequences and abrogate their translation [3].

In eukaryotes, ribosomes that terminally stall for diverse reasons during translation are detected by ribosomeassociated quality control (RQC) (reviewed in $[4,5]$ ). Upon splitting of the $60 \mathrm{~S}$ and $40 \mathrm{~S}$ ribosomal subunits, the RQC complex assembles on the $60 \mathrm{~S}$ subunit to initiate the release and rapid degradation of the truncated tRNA-bound polypeptide. The E3 ubiquitin ligase Listerin (LTN1) modifies the truncated polypeptide with K48-linked ubiquitin chains to target it for degradation in a p97-dependent manner through the proteasome $[3,6,7]$. Whereas peptide release and ribosome recycling by the RQC complex are relatively well understood, less is known about the mechanisms that promote poly(A) recognition and initial ribosome stalling.

(c) The Author(s). 2019 Open Access This article is distributed under the terms of the Creative Commons Attribution 4.0 International License (http://creativecommons.org/licenses/by/4.0/), which permits unrestricted use, distribution, and reproduction in any medium, provided you give appropriate credit to the original author(s) and the source, provide a link to the Creative Commons license, and indicate if changes were made. The Creative Commons Public Domain Dedication waiver (http://creativecommons.org/publicdomain/zero/1.0/) applies to the data made available in this article, unless otherwise stated. 
Several recent studies demonstrated a role for the RNA-binding E3 ubiquitin ligase ZNF598 in initiating RQC for prematurely polyadenylated mRNAs [8-10]. It was suggested that ZNF598 senses the translation of poly(A) segments through binding of the cognate lysine tRNAs [9]. In addition, ZNF598 recognizes the collided di-ribosome structure that arises when a trailing ribosome encounters a slower leading ribosome [11]. This is followed by site-specific, regulatory ubiquitylation of the 40S ribosomal proteins RPS10 and RPS20 by ZNF598. In addition to ZNF598, the 40S ribosomal subunit-associated protein RACK1 was shown to regulate ubiquitylation of RPS2 and RPS3 upstream of ribosomal rescue [10].

Makorin Ring Finger Protein 1 (MKRN1) belongs to a family of evolutionary conserved RNA-binding E3 ubiquitin ligases. Up to four paralogs exist in vertebrates (MKRN1-4), which combine a RING domain with one or more $\mathrm{CCCH}$ zinc finger domains [12, 13] (Additional file 1: Figure S1). MKRN1 has been implicated in the regulation of telomere length, RNA polymerase II transcription, and the turnover of tumor suppressor protein p53 and cell cycle regulator p21 [14-17], but its RNArelated functions remain poorly understood. A study in mouse embryonic stem cells (mESC) reported its interaction with hundreds of mRNAs as well as multiple RNA-binding proteins (RBPs), including the cytoplasmic poly(A)-binding protein (PABP) PABPC1, IGF2BP1, and ELAVL1 [18]. The interaction with the poly(A)-binding protein was further corroborated in human HEK293 cells [19]. The same study demonstrated that a shortened isoform of MKRN1 controls local translation via its PABP-interacting motif 2 (PAM2 motif) in rat neurons [19]. In line with a role in translation, MKRN1 was found in association with ribosomes, from which it could be released together with PABP and other proteins by RNase digestion [20].

The presence of several RNA binding domains and a RING domain in MKRN1 prompted us to study the function of MKRN1 in human cells. Here, we find that MKRN1 is a novel factor in RQC. We show that MKRN1 is recruited to A-rich sequences in mRNAs in a PABPC1-dependent manner. MKRN1 depletion abrogates ribosome stalling at A-rich sequences and results in reduced ubiquitylation of RPS10 and PABPC1. We therefore propose that MKRN1 acts as a first line of defense against poly(A) translation at the mRNA level to prevent premature polyadenylation and the production of erroneous proteins.

\section{Results}

\section{MKRN1 interacts with PABPC1 and other RBPs}

In order to learn about potential functions, we characterized the protein interaction profile of MKRN1 in HEK293T cells. To this end, we used affinity purification
(AP) coupled to stable isotope labelling with amino acids in cell culture (SILAC)-based quantitative mass spectrometry (MS) using GFP-MKRN1 ${ }^{\text {wt }}$ or GFP as a bait. We identified 53 proteins that were significantly enriched in GFP-MKRN1 ${ }^{\text {wt }}$ compared to the control APs (false discovery rate $[\mathrm{FDR}]<5 \%$, combined ratios of three independent experiments). Almost all identified interactors were previously found in association with polyadenylated transcripts (Gene Ontology [GO] term "poly(A) RNA binding," Additional file 1: Figure S2A).

In line with previous reports $[18,19,21]$, we found that MKRN1 strongly interacts with the cytoplasmic poly(A)-binding proteins PABPC1 and PABPC4 (Fig. 1a, Additional file 1: Figure S2B, Additional file 2: Table S1). In addition, we detected 14 ribosomal proteins as well as four proteins that were previously shown to co-purify with ribosomes [20], including IGF2BP1, LARP1, UPF1, and ELAVL1 (Fig. 1a). We confirmed the MS results in reciprocal AP experiments with GFP-tagged PABPC1, ELAVL1, and IGF2BP1 as baits followed by Western blot for endogenous MKRN1 (Additional file 1: Figure S2C and Additional file 3: Figure S10). All detected interactions persisted in the presence of RNases (RNase A and T1), demonstrating that MKRN1 interacts with these proteins in an RNA-independent manner (Additional file 1: Figure S2C). The association is further supported by a study on the Mkrn1 ortholog in Drosophila melanogaster, which identified pAbp, Larp, Upf1, and Imp (IGF2BP in mammals) as interaction partners (Dold et al., bioRxiv, https://doi.org/10.1101/501643).

Our results suggest that MKRN1 is part of a larger mRNA ribonucleoprotein particle (mRNP) together with PABPC1 and other RBPs that are involved in translational regulation. "Nonsense-mediated decay" and "translation" were the most significantly enriched GO terms for the MKRN1 interaction partners (Biological Process, Additional file 1: Figure S2A). Furthermore, MKRN1 was clearly present in polysomal fractions, as determined in sucrose gradient centrifugation experiments. Here, it co-sedimented with PABPC1 (Fig. 1b), indicating that together with PABPC1, MKRN1 is associated with translating ribosomes. Several proteins interact with PABPC1 via a PABP-interacting motif 2 (PAM2) motif, which specifically binds to the MLLE domain present almost exclusively in PABP proteins [23, 24]. Accordingly, a previous study demonstrated that MKRN1 associates with PABP via a PAM2 motif at amino acid positions 161-193 [19]. In support of a putative functional relevance, a phylogenetic analysis illustrated that the presence and positioning of the PAM2 motif are preserved in MKRN1 orthologs across metazoans (Additional file 1: Figure S1A,B). Point mutations in the PAM2 motif of MKRN1 (GFP-MKRN1 ${ }^{\text {PAM2mut }}$ ) [25] strongly compromised its interaction with PABPC1 and PABPC4 in AP experiments followed by MS or Western blot (Fig. 1c-e and 


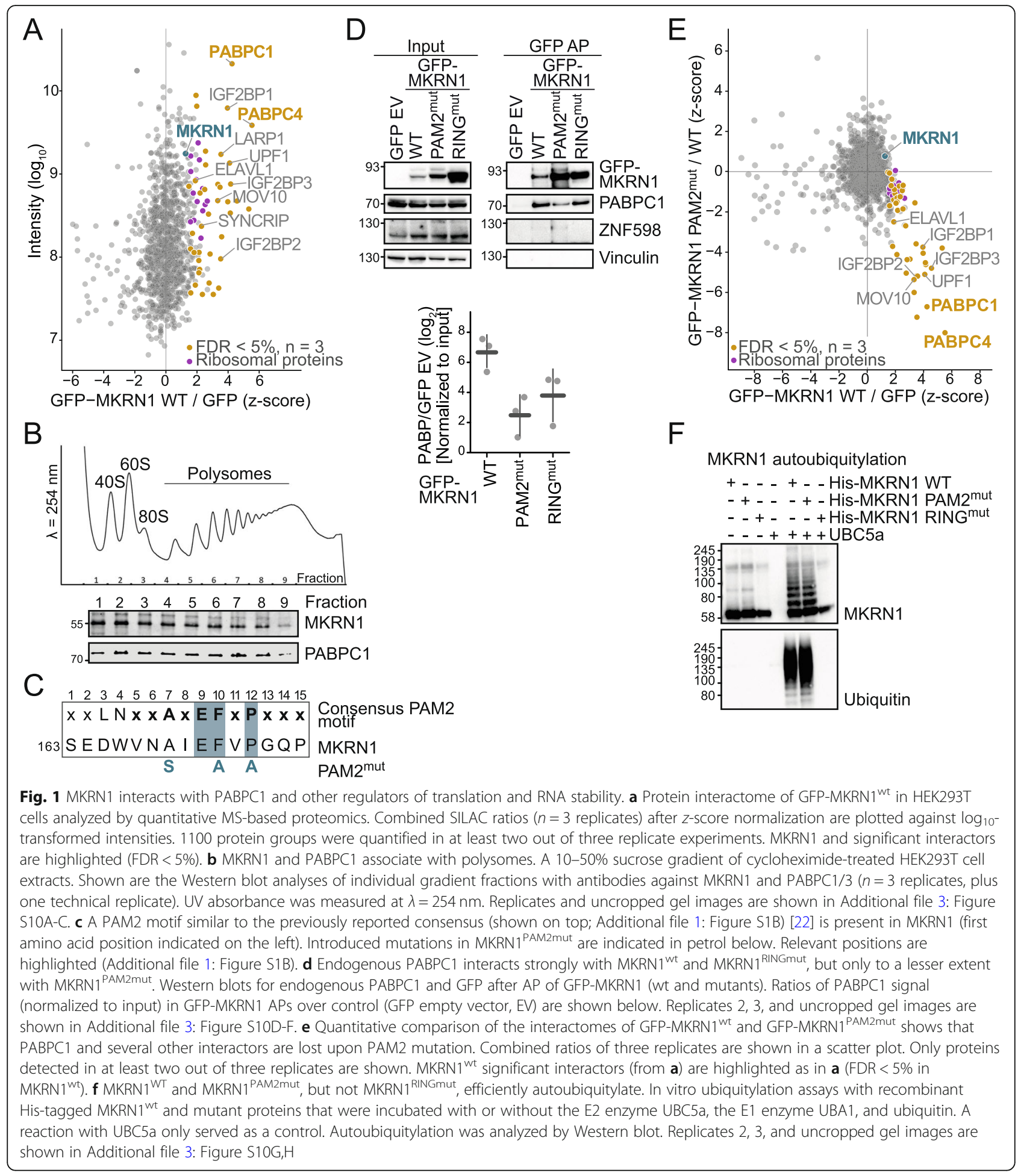

Additional file 2: Table S1). Surprisingly, MKRN1 ${ }^{\text {PAM2mut }}$ lost interaction not only with PABPC1 and PABPC4, but also with several other identified proteins (Fig. 1e), suggesting that the mutant no longer resided within the mRNPs. For comparison, we also tested a previously described point mutation in the RING domain that abolishes the E3 ubiquitin ligase function (ligase-dead, GFP-MKRN1 $1^{\text {RINGmut }}$ ) [14]. This mutant exhibited stable interactions with PABPC1 and other interactors in the AP-MS data. In Western blot experiments, we noted a reduced interaction of MKRN1 ${ }^{\text {RINGmut }}$ with PABPC1. A possible explanation could be the substantially increased levels of MKRN1 $1^{\text {RINGmut in }}$ 
the cells that may skew the normalization in the Western blot experiments (Fig. 1d, Additional file 1: Figure S3 and Additional file 2: Table S1).

In order to rule out that a general loss of protein integrity of MKRN1 ${ }^{\text {PAM2mut }}$, e.g., due to misfolding, is responsible for the observed loss of PABPC1 interaction, we tested the activity of recombinant MKRN1 protein variants in in vitro ubiquitylation assays. As expected, recombinant MKRN1 $1^{\text {RINGmut }}$ was enzymatically inactive, as reflected in the complete absence of autoubiquitylation (Fig. 1f, Additional file 1: Figure S1E). In contrast, both MKRN1 ${ }^{\text {wt }}$ and MKRN1 ${ }^{\text {PAM2mut }}$ could autoubiquitylate with the same efficiency, evidencing that the E3 ubiquitin ligase domain of MKRN1 $1^{\text {PAM2mut }}$ is still functional. Moreover, confocal microscopy of GFP-MKRN $1^{\text {wt }}$ and GFP-MKRN1 ${ }^{\text {PAM2mut }}$ confirmed that the PAM2 mutation did not lead to aggregation nor otherwise impaired protein localization (Additional file 1: Figure S1D). Together, this provides strong evidence that MKRN1 ${ }^{\text {PAM2- }}$ mut is not generally corrupted.

Overall, our results confirm that MKRN1 interacts with PABPC1 and suggest that both proteins associate with translating ribosomes. Loss of the MKRN1PABPC1 interaction impairs mRNP formation, indicating that PABPC1 may be involved in recruiting MKRN1 to the mRNPs.

MKRN1 binds to poly(A) tails and A-rich stretches in 3' UTRs In order to characterize the RNA binding behavior of human MKRN1 in vivo, we performed individual-nucleotide resolution UV crosslinking and immunoprecipitation (iCLIP) [26] in combination with 4-thiouridine (4SU) labeling to enhance UV crosslinking [27]. In three replicate experiments with GFP-tagged MKRN1 (GFP-MKRN1 ${ }^{\text {wt }}$ ) expressed in HEK293T cells, we identified more than 4.6 million unique crosslink events, cumulating into 7331 MKRN1 binding sites (see the "Materials and methods" section; Additional file 1: Figure S4A and Table S2). These were further ranked according to the strength of MKRN1 binding, which was estimated from the enrichment of crosslink events within a binding site relative to its local surrounding as a proxy for transcript abundance ("signalover-background," SOB; see the "Materials and methods" section) [28]. SOB values were highly reproducible between replicates (Pearson correlation coefficients $r>0.72$, Additional file 1: Figure S4B-G).

Across the transcriptome, MKRN1 almost exclusively bound to protein-coding mRNAs with a strong tendency to locate in 3' UTRs (Fig. 2a, b). Binding sites generally harbored uridine-rich tetramers (Additional file 1: Figure S5A), likely reflecting 4SU-based UV crosslinking [27]. Strikingly, the top 20\% MKRN1 binding sites were massively enriched in AAAA tetramers (A, adenosine) within 5-50 nucleotides (nt) downstream of the binding sites
(Fig. 2c and Additional file 1: Figure S5A). The AAAA enrichment reflected the presence of A-rich stretches, which ranged from 8 to $30 \mathrm{nt}$ in length (Additional file 1: Figure S5B; see the "Materials and methods" section). Within 3' UTRs, 30\% (1848 out of 6165) of MKRN1 binding sites resided immediately upstream of an A-rich stretch (Fig. 2a, d) and longer A-rich stretches associated with stronger MKRN1 binding (Additional file 1: Figure S5C,D). Intriguingly, we detected a requirement for a run of at least 8 continuous A's to confer strong MKRN1 binding (Fig. 2e), which precisely matched the RNA footprint of one RNA recognition motif (RRM) domain of PABP [29]. Since PABPC1 was previously reported to bind not only at poly(A) tails but also within 3' UTRs [30-32], these observations suggested that MKRN1 binds together with PABPC1 to mRNAs.

Prompted by this notion, we examined the unusually high fraction of unmapped iCLIP reads in the MKRN1 dataset for untemplated trailing A's to test the hypothesis that MKRN1 may also bind at poly(A) tails (Additional file 1: Table S2). The unmapped reads generally displayed an increased A-content (Fig. 3a), compared to an unrelated control RBP [33], and $6 \%$ of the reads ended in at least ten terminal A's (Fig. 3a, inset). In addition, the mapped GFP-MKRN1 ${ }^{\text {wt }}$ crosslink events were enriched upstream of annotated polyadenylation sites, as exemplified in the SRSF4 gene (Fig. 3b, c). We compared this binding pattern to other RBPs using publicly available eCLIP data from the ENCODE project [34]. The binding of TIAL1, PUM1, QKI, UPF1, and HNRNPK, which are known to fulfill different functions in the 3' UTR [35-38], was distributed throughout 3' UTR bodies (Fig. 3b). In contrast, PABPC4 as well as CPSF6, a component of the cleavage and polyadenylation machinery, peaked together with MKRN1 towards the polyadenylation sites. Together, these results support that MKRN1, but not other 3' UTRbinding proteins, binds at poly(A) tails, where it coincides with the poly(A)-binding protein.

In order to test whether the interaction with PABPC1 influences MKRN1 RNA binding, we performed UV crosslinking experiments with GFP-MKRN1 ${ }^{\text {PAM2mut, }}$ which no longer interacts with PABPC1 (Fig. 1d, e). Strikingly, RNA binding of this mutant was globally reduced compared to GFP-MKRN1 ${ }^{\text {wt }}$ (Fig. 3d, Additional file 1: Figure S6A,B, and Additional file 3: Figure S11), indicating that PABPC1 might recruit MKRN1 to RNA. In order to substantiate this finding, we performed in vitro RNA pulldown assays with recombinant HisPABPC1 and/or His-MKRN1 and biotinylated RNAs. Notably, addition of His-PABPC1 strongly increased the pulldown of His-MKRN1 with the $\mathrm{A}_{20}$-harboring RNA but not the control RNA (Fig. 3e and Additional file 1: Figure S6C,D), indicating that PABPC1 stabilizes MKRN1 at poly(A) sequences. 


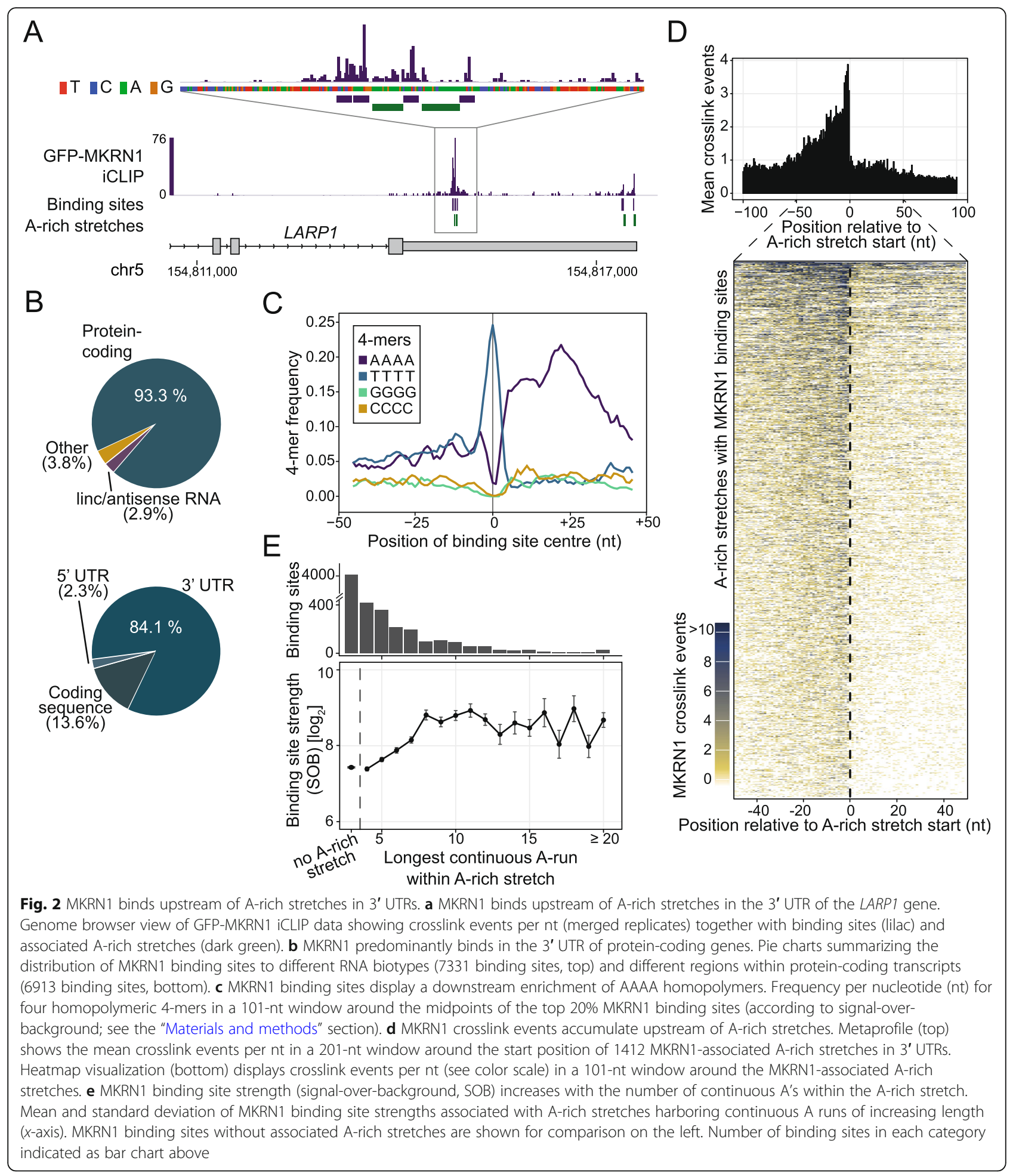

In summary, these results strongly suggest that MKRN1 binds upstream of A-rich stretches in 3' UTRs and poly(A) tails. We hypothesize that this binding pattern is defined via the interaction of MKRN1 with PABPC1, since (i) MKRN1 binding intensifies when the associated A-rich stretch reaches sufficient length to accommodate one RRM domain of PABPC1, (ii) abolishing the MKRN1-PABPC1 interaction results in loss of MKRN1 RNA binding, and (iii) PABPC1 reinforces MKRN1's association with RNA in vitro. In a concordant scenario, it was found that Drosophila Mkrn1 binds before an 


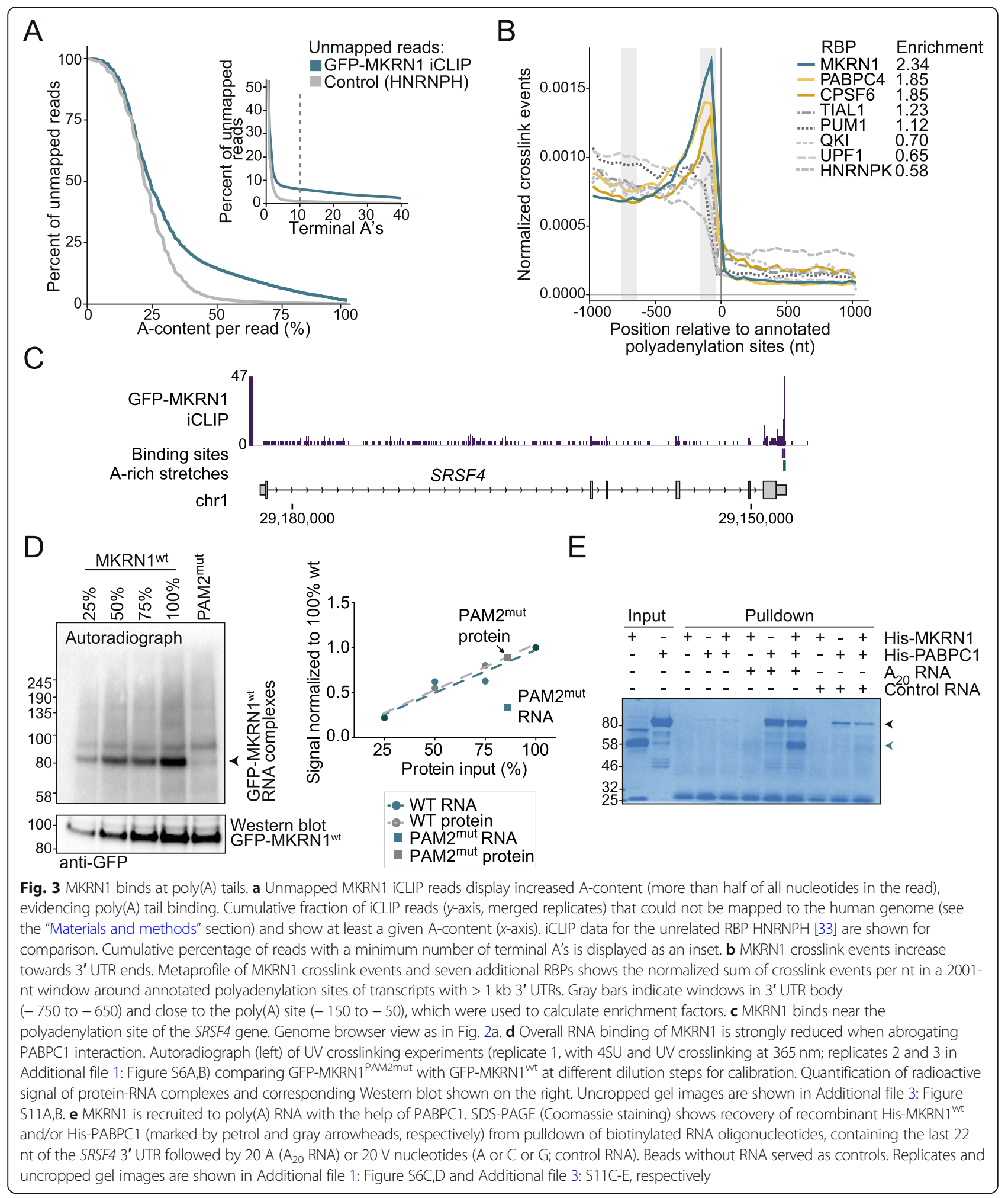

extended A-rich stretch in the 3' UTR of oskar mRNA and that this binding is significantly reduced upon depletion of pAbp (Dold et al., bioRxiv, https:// doi.org/10.1101/501643).
MKRN1 promotes ribosome stalling at poly(A) sequences As outlined above, our iCLIP data demonstrated that MKRN1 marks the beginning of poly(A) tails. Hence, it is conceivable that MKRN1 will also bind upstream of 
premature polyadenylation events within open reading frames. Based on MKRN1's binding pattern, its interaction partners, and its association with ribosomes, we hypothesized that MKRN1 may be involved in the clearance of such transcripts by ribosome-associated quality control (RQC). In this process, ribosomes that translate into a poly(A) sequence, for instance upon stop codon readthrough or premature polyadenylation, are stalled and eventually recycled $[4,5]$. To test this hypothesis, we employed a recently introduced flow cytometrybased assay that monitors ribosome stalling in a dual fluorescence reporter $[8,10]$. In this reporter, the genes encoding the green and red fluorescent protein (GFP and RFP, respectively) are separated by a linker region with a poly(A) stretch as putative ribosome stalling sequence (Fig. 4a). The linker is flanked by two viral P2A sites that promote translational elongation into separate protein products, thereby disconnecting the translation of the linker region from the flanking GFP and RFP translation products [39]. As a consequence, complete translation of the reporter results in equal amounts of three stand-alone proteins (GFP, linker peptide, and RFP), whereas abortion of translation within the linker sequence impairs RFP, but not GFP production. This results in a reduced RFP:GFP ratio [8], which is measured using fluorescence-based flow cytometry.

As reported previously, inserting a $\mathrm{K}(\mathrm{AAA})_{20}$ linker (encoding 20 lysine residues) into the reporter resulted in predominant ribosome stalling compared to the starting vector $\left(\mathrm{K}_{0}\right.$, Fig. $4 \mathrm{~b}$ and Additional file 1: Figure S7). Importantly, MKRN1 depletion with two independent siRNAs led to a reproducible recovery of RFP expression downstream of $\mathrm{K}(\mathrm{AAA})_{20}$, suggesting that many ribosomes failed to stall at K(AAA) $)_{20}(M K R N 1 \mathrm{KD} 1$ and KD2; Fig. 4b, Additional file 1: Figure S7A, S8A,B, and Additional file 3: Figure S12). MKRN1 KD2 seemed slightly more effective, possibly because this siRNA simultaneously decreased the transcript levels of the close paralog MKRN2 (Additional file 1: Figure S8B). The specific impact of MKRN1 on ribosome stalling was further supported by complementing the MKRN1 KD with stable integration of MKRN1 constructs. Only MKRN1 $1^{\mathrm{wt}}$

A
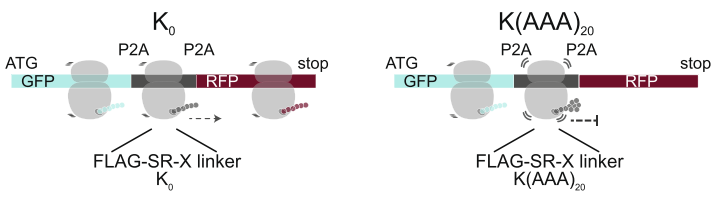

B

C
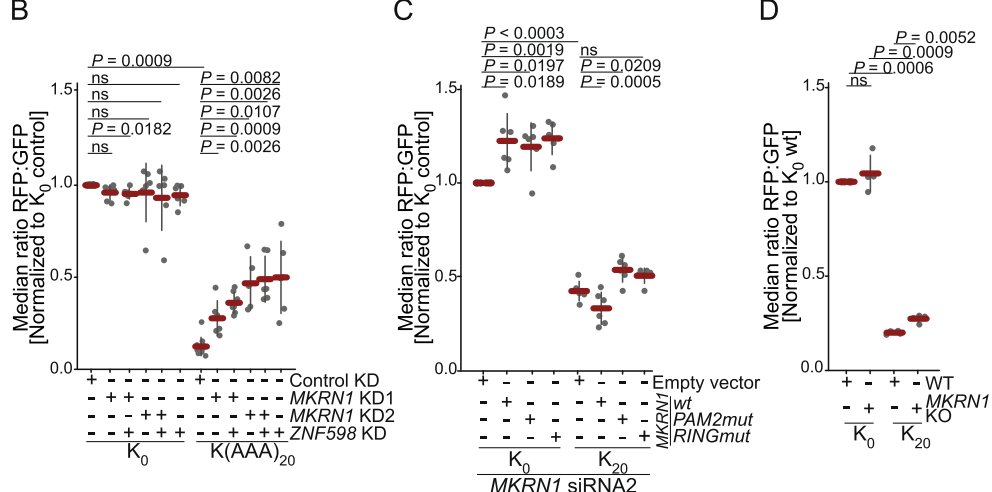

Fig. 4 MKRN1 stalls ribosomes at poly(A) sequences. a The dual fluorescence reporter harbors an N-terminal GFP, followed by a FLAG-SR-X linker and a C-terminal RFP, which are separated by P2A sites to ensure translation into three separate proteins [8]. The resulting GFP:RFP ratio was determined using flow cytometry. The inserted fragment K(AAA) 20 encodes 20 lysines by repeating the codon AAA. The starting vector without insert $\left(K_{0}\right)$ served as control. Schematic ribosomes illustrate translation of the respective reporter segments. b Ribosomes fail to stall in the absence of MKRN1. HEK293T cells were transfected with control siRNA or siRNAs targeting MKRN1 (KD1 and KD2) or ZNF598 for $24 \mathrm{~h}$, followed by transfection of the reporter plasmids for $48 \mathrm{~h}$. Western blots for KDs are shown in Additional file 1: Figure S8A. RFP and GFP signals were analyzed by flow cytometry. Median RFP:GFP ratios, normalized to $K_{0}$ in control, are shown. Error bars represent s.d.m.; $P$ values indicated above (paired two-tailed Student's $t$ test, Benjamini-Hochberg correction, $n \geq 6$ replicates; ns, not significant). Analyses for inserts coding for 12 lysines (K(AAA) $\left.)_{12}\right)$ and ten arginines $\left(R(C G A)_{10}\right)$ in the dual fluorescence reporter are shown in Additional file 1: Figure S7A. c Expression of MKRN1 ${ }^{\text {wt }}$ can rescue ribosome stalling. HEK293T cell lines with stable integrations of siRNA2-insensitive MKRN1 wild type and mutant constructs, or empty vector, were transfected with MKRN1 siRNA2 for $24 \mathrm{~h}$, followed by transfection of the reporter plasmids for $48 \mathrm{~h}$. RFP and GFP signals were analyzed by flow cytometry. Median RFP:GFP ratios, normalized to $K_{0}$ in WT cells, are shown. Error bars represent s.d.m.; $P$ values indicated above (paired two-tailed Student's $t$ test, Benjamini-Hochberg correction, $n=6$ replicates; ns, not significant). Analyses for reporter plasmids with inserts coding for $\mathrm{K}(\mathrm{AAA})_{12}$ or $\mathrm{R}(\mathrm{CGA})_{10}$ are shown in Additional file 1: Figure S7B. d MKRN1 knockout (MKRN1 KO) and wild type (WT) HEK293T cells were transfected with the reporter plasmids for $48 \mathrm{~h}$. Measurements, analyses, and visualization as in $\mathbf{c}(n=4$ replicates). Analyses for reporter plasmids with inserts coding for $\mathrm{K}(\mathrm{AAA})_{12}$ or $\mathrm{R}(\mathrm{CGA})_{10}$ are shown in Additional file 1: Figure $\mathrm{S} 7 \mathrm{C}(\mathbf{d})$ 
was capable of partially reverting the KD effect, such that ribosome stalling was partially restored, whereas neither of the MKRN1 mutants was functional in this assay (Fig. 4c and Additional file 1: Figure S7B). In addition, we found that MKRN1 KD2 did not influence the abundance of the underlying reporter RNA (Additional file 1: Figure S7D), indicating that $\mathrm{RQC}$ for this reporter is not coupled to appreciable mRNA destabilization in HEK293T cells, in line with a previous study [8].

In order to relate MKRN1's effect to other players of the RQC pathway, we knocked down ZNF598, the E3 ubiquitin ligase that was recently reported to stall ribosomes during RQC [8-10]. Importantly, MKRN1 KD2 impaired ribosome stalling to a similar extent as KD of ZNF598. Simultaneous depletion of MKRN1 and ZNF598 was not additive, indicating that both proteins work in the same pathway (Fig. 4b and Additional file 1: Figure S7A). We could not detect an interaction between MKRN1 and ZNF598 in pulldown experiments (Fig. 1d). However, we noted a certain level of cross-regulation, such that ZNF598 expression was decreased in MKRN1 KD1 (but not in MKRN1 KD2), whereas ZNF598 overexpression reduced MKRN1 expression (Additional file 1: Figure S8). Overall, these results suggest that MKRN1 fulfills a specific function in RQC complementary to ZNF598.

For independent validation of the $\mathrm{KD}$ results, we generated a stable MKRN1 knockout (KO) cell line using CRISPR/Cas9 genome editing (Additional file 1: Figure S8C,D). Reporter assays showed that complete loss of MKRN1 impaired ribosome stalling at $\mathrm{K}(\mathrm{AAA})_{20}$, albeit with a smaller effect size compared to the siRNAmediated KD (Fig. 4d and Additional file 1: Figure S7C). qPCR indicated a compensatory upregulation of the paralog MKRN2 in the MKRN1 KO but not in the MKRN1 KD (Additional file 1: Figure S8B,D), which could explain the reduced effect size of the MKRN1 KO in the reporter assays. In line with a partially redundant role of MKRN2, we find that simultaneous depletion of MKRN1 and MKRN2, as observed upon KD with siRNA2 (Additional file 1: Figure S8B), shows a larger effect than KD with siRNA1, which does not change MKRN2 levels.

Based on these results, we propose MKRN1 as a novel player in RQC that contributes to efficient ribosome stalling at poly(A) sequences. Our experiments suggest that this function likely depends on the interaction of MKRN1 with PABPC1 as well as its E3 ubiquitin ligase activity.

MKRN1 mediates the ubiquitylation of RPS10 and PABPC1 RQC builds on a series of ubiquitylation events by multiple E3 ubiquitin ligases, including Listerin and ZNF598 [5]. In order to identify putative ubiquitylation substrates of MKRN1, we performed ubiquitin remnant profiling to compare the relative abundance of di-glycine-modified lysines in MKRN1 KD and control cells. We quantified 2324 ubiquitylation sites (in 1264 proteins) that were detected in all four replicate experiments (Additional file 4: Table S3). Notably, MKRN1 depletion led to a significantly decreased abundance of 29 ubiquitylation sites on 21 proteins $($ FDR $<10 \%$, Fig. 5a). Comparing the relative abundance of putative substrates in total extracts showed that none of them significantly changed in the MKRN1 KD cells, suggesting that ubiquitylation does not trigger substantial degradation of these proteins (Additional file 1: Figure S9A and Additional file 5: Table S4). This result was further confirmed by Western blot for several proteins that were identified as putative MKRN1 substrates (Additional file 1: Figure S9B).

The majority of the ubiquitylation targets formed a functional cluster of translational regulators based on our own interactome data, previously reported proteinprotein interactions, and functional annotations (Fig. 5b and Additional file 1: Figure S9C). Intriguingly, we identified a MKRN1-dependent ubiquitylation site on RPS10, a $40 \mathrm{~S}$ ribosomal protein that was previously reported to be modified by ZNF598 during RQC [8-10]. Here, MKRN1 KD led to a significant decrease in ubiquitylation at lysine 107 of RPS10 (K107; Fig. 5c-f). In addition, MKRN1 KD decreased ubiquitylation of a number of interaction partners including PABPC1/4, IGF2BP1/2/3, LARP1, MOV10, and ELAVL1 (Fig. 5d). In order to test whether MKRN1 can directly modify RPS10 and PABPC1, we performed in vitro ubiquitylation assays with recombinant proteins. Western blot experiments detected the appearance of multiple mono- and poly-ubiquitylated variants of RPS10 and PABPC1, illustrating that MKRN1 efficiently ubiquitylated both proteins (Fig. $5 \mathrm{~g}$ ). We therefore propose that rather than recruiting ZNF598 or another E3 ubiquitin ligase, MKRN1 plays an active role in RQC by regulatory ubiquitylation of RPS10.

\section{Discussion}

Ribosome-associated quality control is essential to recognize and clear terminally stalled ribosomes. Here, we put forward MKRN1 as a novel factor in RQC. Our data suggest that MKRN1 is positioned upstream of poly(A) sequences through direct interaction with PABPC1, thereby marking the beginning of poly(A) tails. We propose that in case of premature polyadenylation or stop codon read-through, MKRN1 stalls the translating ribosome and initiates RQC by ubiquitylating ribosomal protein RPS10, PABPC1, and other translational regulators (Fig. 6).

Central to our model is the specific RNA binding behavior of MKRN1, which is recruited to mRNA by PABPC1 to mark the beginning of A-rich stretches and poly(A) tails. This hypothesis builds on the strong interaction of both proteins, the reduced RNA binding of MKRN1 when the interaction is abolished in vivo, the 


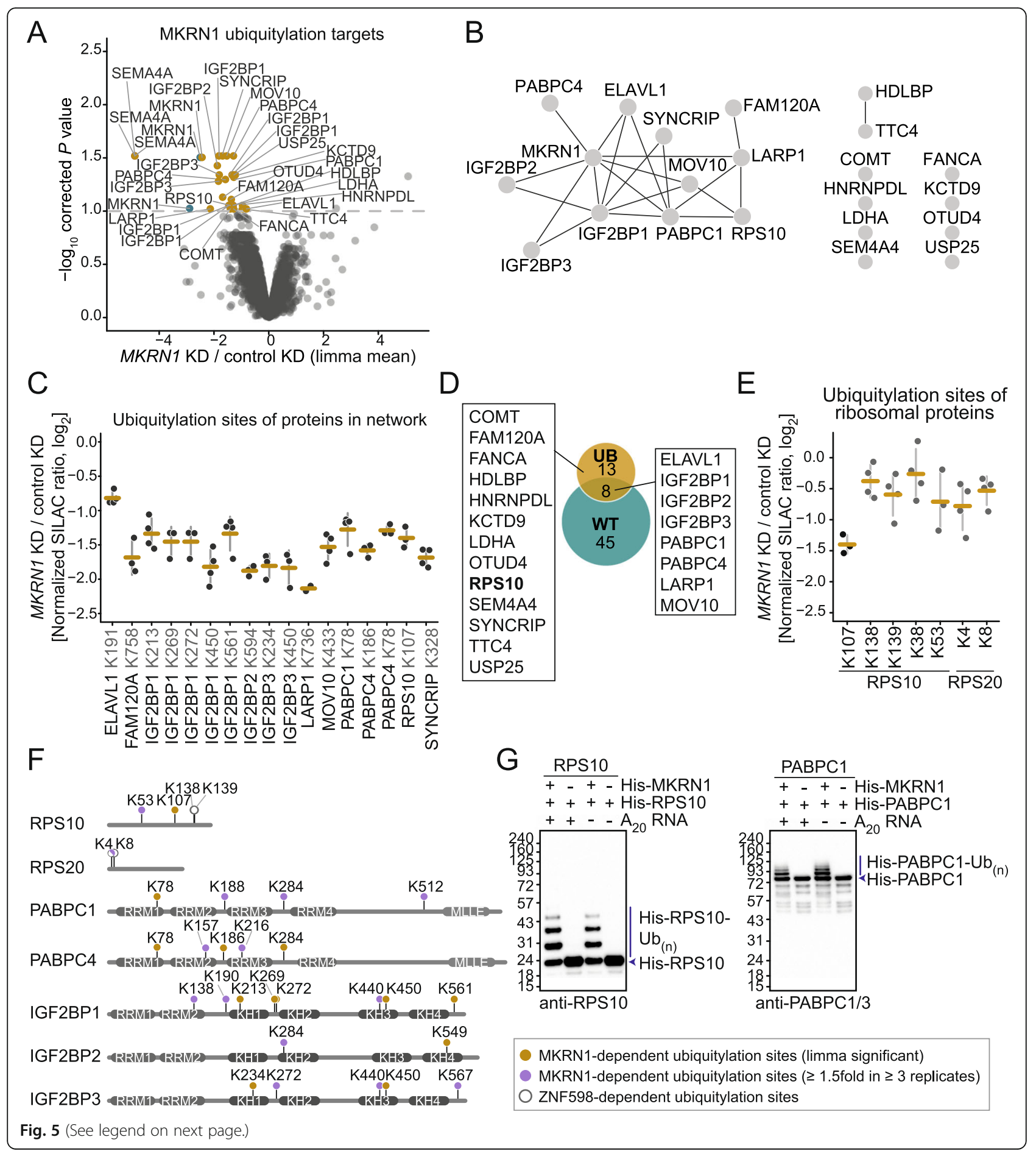


(See figure on previous page.)

Fig. 5 MKRN1 ubiquitylates ribosomal protein RPS10 and translational regulators. a Ubiquitin remnant profiling to compare the relative abundance of ubiquitylation sites in MKRN1 KD2 and control HEK293T cells. Ubiquitin remnant peptides were enriched and analyzed by quantitative mass spectrometry, quantifying a total of 15,528 ubiquitylation sites on 4790 proteins. 29 putative MKRN1 target sites with significantly decreased ubiquitylation upon MKRN1 KD2 (FDR $<10 \%, n=4$ replicates) are highlighted and labeled with the respective protein name. Note that many proteins contain several differentially regulated ubiquitylation sites. b Protein interaction network of 21 proteins with putative MKRN1 ubiquitylation target sites (significantly reduced, shown in a). The functional interactions were obtained from the STRING and BioGrid databases and our study. Visualization by Cytoscape. c Ubiquitin remnant profiling results for significantly regulated ubiquitylation sites $(F D R<10 \%)$ in proteins from network in $\mathbf{b}$. Mean and standard deviation of the mean (s.d.m., error bars) are given together with all data points. d Comparison of interactome of GFP-MKRN1 ${ }^{\text {wt }}$ (WT over GFP, see Fig. 1a) with putative MKRN1 ubiquitylation substrates from ubiquitin remnant profiling (UB, see a). Protein names are given for all ubiquitylation substrates. e Ubiquitin remnant profiling results for seven quantified ubiquitylation sites in RPS10 and RPS20. Significant changes are shown in black (FDR < 10\%) and non-significant changes in gray. Representation as in c. $\mathbf{f}$ Comparison of ubiquitylation sites in the target proteins RPS10 (UniProt ID P46783), RPS20 (P60866), PABPC1 (P11940), PABPC4 (B1ANRO), IGF2BP1 (Q9NZ18), IGF2BP2 (F8W930), and IGF2BP3 (O00425) that are modified by ZNF598 and MKRN1 during RQC. g MKRN1 ubiquitylates RPS10 and PABPC1 in vitro. His-RPS10 (left) or His-PABPC1 (right) were incubated with or without His-MKRN1. Ubiquitylation of the target proteins was assessed by Western blot. Replicates 2, 3, and uncropped gel images are shown in Additional file 3: Figure S121,J for RPS10 and Additional file 3: Figure S12K,L for PABPC1

reinforced RNA binding of MKRN1 in the presence of PABPC1 in vitro, and the occurrence of strong MKRN1 binding when at least 8 continuous A's are present. The latter mirrors the footprint of one RRM domain of PABPC1, indicating that the binding of one RRM of PABPC1 to poly(A) is sufficient for MKRN1 recruitment [29]. Of note, a study with the Mkrn1 ortholog from $D$. melanogaster demonstrates binding of a Mkrn1/pAbp complex at an A-rich stretch in the 3' UTR of oskar mRNA, which is involved in translational control and required for oogenesis (Dold et al., bioRxiv, doi: https:// doi.org/10.1101/501643).

Our data show that MKRN1 associates with polysomes and ubiquitylates RPS10, indicating a role in translational control. We hypothesize that ribosomes encountering the MKRN1-PABPC1 complex are stalled, possibly via ubiquitylation of RPS10 on K107 and other MKRN1 substrates. Concordantly, ZNF598 was also found to mediate ubiquitylation of RPS10 on K138/K139 [11]. In conjunction with the unique RNA binding behavior, we therefore hypothesize that MKRN1 acts as a first line of defense against poly(A) translation. We propose that MKRN1 is recruited by PABPC1 to the beginning of poly(A) tails, including premature polyadenylation events within open reading frames, where it represents a physical "roadblock" to the translating ribosome. Upon contact with the translating ribosome, MKRN1 ubiquitylates K107 on RPS10, thereby stalling the ribosome before it translates the poly(A) tail. Subsequently, the trailing ribosomes collide with the initially stalled ribosome. ZNF598 recognizes the collision interface and ubiquitylates the collided ribosomes $[11,40]$. In summary, we suggest that a sequence of MKRN1-mediated and ZNF598-mediated ubiquitylation events on ribosomal proteins and possibly other factors, including PABPC1, triggers ribosome-associated quality control. The type of ubiquitin chain deposited by MKRN1 on RPS10 as well as the possible existence of deubiquitylating enzymes that cleave the ubiquitin chain from RPS10 remain to be investigated.

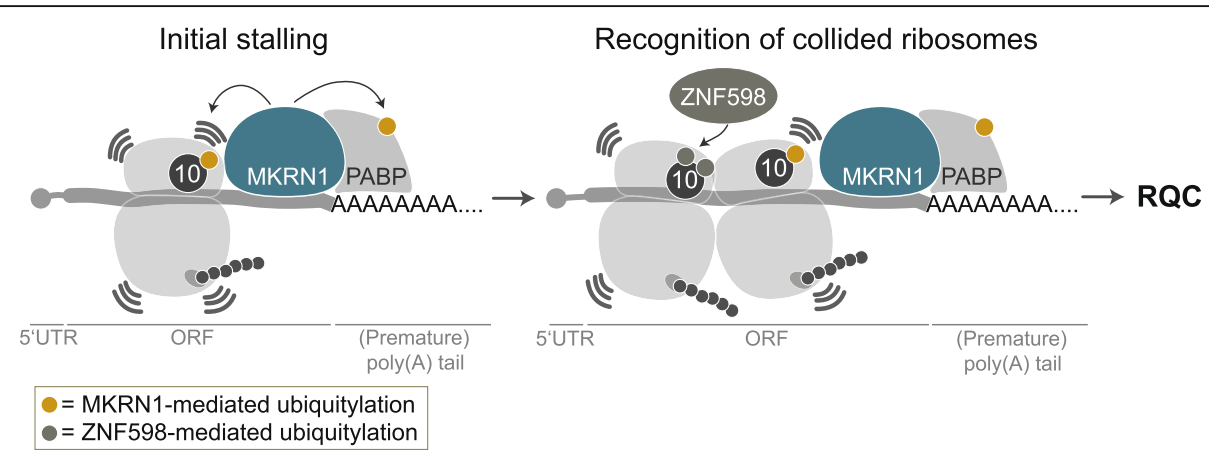

Fig. 6 MKRN1 is a sensor for poly(A) sequences that stalls ribosomes to initiate ribosome-associated quality control. Proposed model of MKRN1 function: MKRN1 is positioned upstream of (premature) poly(A) tails via interaction with PABPC1. Ribosomes translating the open reading frame run into MKRN1 that acts as a roadblock to prohibit poly(A) translation. Upon contact with the translating ribosome, MKRN1 ubiquitylates the 40S ribosomal protein RPS10. This stalls the ribosome, causing the trailing ribosomes to collide. ZNF598 recognizes the collided ribosomes and ubiquitylates ribosomal proteins to promote $R Q C$ 
Many known components of the RQC machinery, such as Listerin (Ltn1p in yeast) and ZNF598 (Hel2p in yeast), are conserved from yeast to human; however, the molecular signals that are recognized partially differ. In yeast, RQC can be triggered by an excess of positively charged amino acids (lysine and arginine), which are sensed while they pass through the ribosomal exit tunnel [41, 42]. In contrast, in human, sensing the aberrant mRNAs does not occur via the encoded amino acids but at the level of the mRNA sequence and corresponding tRNAs, such that only poly(A) effectively results in ribosome stalling [8, 9, 43]. We propose that MKRN1 acts as a direct reader of poly(A) sequences based on its interaction with PABPC1. Consistent with this conceptual difference, there is no functionally equivalent ortholog of MKRN1 in yeast (Yth1p and Lee1p are similar, but lack the RING domain and the PAM2 motif; Additional file 1: Figure S1C). However, a recent study in yeast has shown that Hel2p binds before and also after the stop codon within 3' UTRs of mRNAs [44], while such a binding pattern has not been observed for ZNF598 in human cells [9]. Even though MKRN1 and Hel2p binding patterns are not identical, this suggests the possibility that the roles of yeast Hel2p might have been split between MKRN1 and ZNF598 in human cells.

Why yeast and human employ partially different mechanisms to detect poly(A) translation is currently unclear, but it has been suggested that spurious translation of polylysine stretches from long human poly(A) tails might target the aberrant proteins to the nucleus [8]. Loss of mRNA surveillance and RQC deficiency can lead to protein aggregation and culminate in proteotoxic stress, which in turn is lined to neurological disorders such as amyotrophic lateral sclerosis $[45,46]$. Hence, recognition of poly(A) sequences prior to their translation, possibly by the action of MKRN1, might be particularly beneficial in humans.

\section{Materials and methods Cell culture}

HEK293T cells were obtained from DSMZ and cultured in DMEM (Life Technologies) with 10\% fetal bovine serum (Life Technologies), 1\% penicillin/streptomycin (Life Technologies), and 1\% L-glutamine (Life Technologies). All cells were maintained at $37{ }^{\circ} \mathrm{C}$ in a humidified incubator containing $5 \% \mathrm{CO}_{2}$ and routinely tested for mycoplasma infection. For SILAC labeling, cells were maintained in media containing either L-arginine and L-lysine (light SILAC label), L-arginine $\left({ }^{13} \mathrm{C}_{6}\right)$ and L-lysine $\left({ }^{2} \mathrm{H}_{4}\right)$ (medium SILAC label), or L-arginine $\left({ }^{13} \mathrm{C}_{6}{ }^{15} \mathrm{~N}_{4}\right)$ and L-lysine $\left({ }^{13} \mathrm{C}_{6}{ }^{15} \mathrm{~N}_{2}\right)$ (heavy SILAC label) (Cambridge Isotope Laboratories).

\section{Vectors}

The following vectors, suitable for Gateway Cloning, were obtained either from the IMB Core Facility ORFeome Collection [47] or from the Harvard PlasmID Repository (https://
plasmid.med.harvard.edu/PLASMID/): pENTR221-MKRN1, pENTR221-PABPC1, pENTR223.1-IGF2BP1, pENTR221ELAVL1, and pCMV-SPORT-ZNF598. Coding sequences from the entry vectors were cloned into the mammalian expression vectors pMX-DEST53-IP-GFP by LR Gateway cloning according to the manufacturer's recommendations (Gateway LR Clonase II Enzyme mix; Life Technologies). Dual fluorescence reporter plasmids (pmGFP-P2A-K $\mathrm{K}_{0}$ P2A-RFP, pmGFP-P2A- $\left(\mathrm{K}^{\mathrm{AAA}}\right)_{12}$-P2A-RFP, pmGFP-P2A$\left(\mathrm{K}^{\mathrm{AAA}}\right)_{20}$-P2A-RFP, and pmGFP-P2A-( $\left.{ }^{\mathrm{CGA}}\right)_{10}$-P2A-RFP) were generously provided by Ramanujan S. Hegde (MRC Laboratory of Molecular Biology, Cambridge, UK) [8].

\section{Cloning}

All MKRN1 mutant plasmids were generated with the Q5 Site-Directed Mutagenesis Kit (NEB) according to the manufacturer's recommendations. In order to disrupt MKRN1's interaction with PABPC1 (MKRN1 ${ }^{\text {PAM2mut), }}$ three-point mutations were introduced into the PAM2 motif (A169S, F172A, P174A; Fig. 1c) as previously described [25]. In MKRN1 ${ }^{\text {RINGmut, a previously described }}$ mutation in the RING domain (H307E) was introduced to abolish E3 ubiquitin ligase function [14]. In order to obtain a siRNA2-insensitive copy of MKRN1 for complementation, we used alternative codons and wobble positions where possible to synonymize the nucleotide sequence in the siRNA target site. For untagged MKRN1, GFP was deleted from the pMX-DEST-GFP vector using the Q5 Site-Directed Mutagenesis Kit. All oligonucleotides used for cloning are listed in Additional file 1: Table S5.

\section{Transfections}

Overexpression of vectors was performed using Polyethylenimine MAX 4000 (PEI, Polysciences, 24885-2) with a DNA:PEI ratio of 1:10. Knockdowns were performed with siRNAs (Additional file 1: Table S6) using Lipofectamine RNAiMAX (Life Technologies) according to the manufacturer's recommendations.

\section{Affinity purification (AP) for Western blot analyses}

GFP-based affinity purifications (APs) were performed as described before [21]. In brief, HEK293T cells transiently expressing GFP (empty vector) or a GFP-tagged target protein were used. The cells were lysed in modified RIPA (mRIPA) buffer supplemented with protease inhibitors (protease inhibitor cocktail, Sigma), $1 \mathrm{mM}$ sodium orthovanadate, $5 \mathrm{mM} \beta$-glycerophosphate, $5 \mathrm{mM}$ sodium fluoride, and $10 \mathrm{mM} N$-ethylmaleimide (NEM) (all from Sigma). Protein concentrations were determined using the Pierce BCA Protein Assay Kit (Thermo Fisher). GFP-trap agarose beads (Chromotek) were incubated with the cleared lysate for $1 \mathrm{~h}$ at $4{ }^{\circ} \mathrm{C}$. After five washes with MRIPA buffer, the beads were resuspended 
in LDS sample buffer (Life Technologies) and heated to $70{ }^{\circ} \mathrm{C}$ for $10 \mathrm{~min}$. For RNase digests, the enriched proteins were incubated with $0.5 \mathrm{U} / \mu \mathrm{l}$ RNase A (Qiagen) and $20 \mathrm{U} / \mu \mathrm{l}$ RNase T1 (Thermo Fisher Scientific) for 30 min at $4{ }^{\circ} \mathrm{C}$ after the first two washes in mRIPA buffer.

\section{Sample preparation for the protein interactome analysis} GFP-based APs were performed as described before [21]. In brief, HEK293T cells transiently expressing GFP (empty vector) were cultured in light SILAC medium, while cells expressing $\mathrm{N}$-terminally GFP-tagged MKRN1 wt or mutants were cultured in medium or heavy SILAC medium. The cells were lysed as described above. After washing in mRIPA buffer, GFP-trap agarose beads were incubated with the cleared lysate for $1 \mathrm{~h}$ at $4{ }^{\circ} \mathrm{C}$. All AP samples were washed four times with mRIPA buffer, combined and washed again in mRIPA buffer. The beads were heated in LDS sample buffer, supplemented with 1 $\mathrm{mM}$ dithiothreitol (DTT; Sigma, D5545) for $10 \mathrm{~min}$ at $70^{\circ} \mathrm{C}$ and alkylated using $5.5 \mathrm{mM}$ 2-chloroacetamide (CAA; Sigma, C0267) for 30 min at RT in the dark [48].

\section{Sample preparation for the proteome analysis}

$M K R N 1 \mathrm{KD}$ using siRNA2 was performed in heavy labeled SILAC cells, and control KD was performed in light labeled SILAC cells in two replicates. For the third replicate, a label swop was performed, knocking down MKRN1 (siRNA2) in light labeled SILAC cells and control in heavy labeled SILAC cells. For proteome analysis, cells were lysed as described above. Subsequently, $25 \mu \mathrm{g}$ protein from each SILAC condition $(50 \mu \mathrm{g}$ in total) were pooled and processed as described below.

\section{Sample preparation for mass spectrometry}

The enriched proteins were resolved by SDS-PAGE on a NuPAGE 4-12\% Bis-Tris protein gel (Thermo Fisher Scientific) and stained using the Colloidal Blue Staining Kit (Life Technologies). Proteins were in-gel digested using trypsin, before peptides were extracted from the gel. To concentrate, clear, and acidify the peptides, they were bound to C18 StageTips as described previously [49].

\section{Mass spectrometry data acquisition}

Peptide fractions were analyzed on a quadrupole Orbitrap mass spectrometer (Thermo Q Exactive Plus, Thermo Scientific) coupled to an uHPLC system (EASY-nLC 1000, Thermo Scientific) [50]. Peptide samples were separated on a $\mathrm{C} 18$ reversed phase column (length $20 \mathrm{~cm}$, inner diameter $75 \mu \mathrm{m}$, bead size $1.9 \mu \mathrm{m}$ ) and eluted in a linear gradient from 8 to $40 \%$ acetonitrile containing $0.1 \%$ formic acid in $105 \mathrm{~min}$ for the interactome analyses, in $175 \mathrm{~min}$ for the proteome analyses, or in $125 \mathrm{~min}$ for the ubiquitylome analyses. The mass spectrometer was operated in data-dependent positive mode, automatically switching between MS and $\mathrm{MS}^{2}$ acquisition. The full scan MS spectra $(\mathrm{m} / \mathrm{z} 300-1650)$ were acquired in the Orbitrap. Sequential isolation and fragmentation of the ten most abundant ions were performed by higher-energy collisional dissociation (HCD) [51]. Peptides with unassigned charge states, as well as with charge states less than +2 were excluded from fragmentation. The Orbitrap mass analyzer was used for acquisition of fragment spectra.

\section{Peptide identification and quantification}

Raw data files were analyzed and peptides were identified using the MaxQuant software (version 1.5.28) [52]. Parent ion and $\mathrm{MS}^{2}$ spectra were compared to a database containing 92,578 human protein sequences obtained from UniProtKB (release June 2018), coupled to the Andromeda search engine [53]. Cysteine carbamidomethylation was set as a fixed modification. N-terminal acetylation, oxidation, and $\mathrm{N}$-ethylmaleimide (NEM) were set as variable modifications. For ubiquitylome data analysis, glycine-glycine (GlyGly) modification of lysine was additionally set as a variable modification. The mass tolerance for the spectra search was set to be lower than $6 \mathrm{ppm}$ in MS and $20 \mathrm{ppm}$ in HCD $\mathrm{MS}^{2}$ mode. Spectra were searched with strict trypsin specificity and allowing for up to three mis-cleavages. Site localization probabilities were determined by MaxQuant using the PTM scoring algorithm as described previously $[54,55]$. Filtering of the dataset was based on the posterior error probability to arrive at a false discovery rate (FDR) < $1 \%$ estimated using a target-decoy approach. Proteins that were categorized as "only identified by site", potential contaminants and reverse hits were removed. Only proteins identified with at least two peptides (including at least one unique peptide) and a SILAC ratio count of at least two were used for analysis. For AP experiments, proteins that were quantified in at least two out of three experiments were kept for further analysis. In total, we quantified 1106 and 1097 protein groups in the AP experiments with GFPMKRN1 ${ }^{\text {wt }}$ (Fig. 1a), GFP-MKRN1 ${ }^{\text {PAM2mut }}$ (Fig. 1e) and GFP-MKRN1 ${ }^{\text {RINGmut }}$ (Additional file 1: Figure S3), respectively (Additional file 2: Table S1). The SILAC ratios were $\log _{2}$-transformed and converted into an asymmetric $z$ score based on the mean and interquartile range of the distribution as described previously [54]. For statistical analysis, a moderated $\mathrm{t}$-test from the limma algorithm was used [56]. Enriched proteins with an FDR $<5 \%$ were determined to be significantly enriched interactors (for GFP$M K R N 1^{\text {wt }}$ ). For proteins enriched in GFP-MKRN $1^{\text {RINGmut }}$ over GFP-MKRN1 $1^{\text {wt }}$, proteins with an $\mathrm{FDR}<5 \%$ and a GFP-MKRN $1^{\text {wt }} /$ GFP $z$-score $>1$ were selected. In the proteome experiment, we quantified 6439 protein groups, present in all three replicates. Ratio-ratio and ratiointensity plots were created in $\mathrm{R}$ (version 3.4.3) using RStudio (http://www.rstudio.com/). 


\section{Functional annotation of target proteins}

In order to assess the functions of MKRN1-interacting proteins and proteins with MKRN1-dependent ubiquitylation sites, we performed gene ontology (GO) enrichment analyses using the Database for Annotation, Visualization and Integrated Discovery (DAVID 6.7) for three GO domains [57]. Enriched GO terms (modified Fisher exact test, adjusted $P$ value $<0.05$, BenjaminiHochberg correction; Additional file 1: Figure S2A and Figure S9C) were visualized using REVIGO (Reduce \& Visualize Gene Ontology) allowing medium GO term similarity [58].

\section{Western blot}

Denatured proteins were separated by SDS-PAGE on a NuPAGE 4-12\% Bis-Tris protein gel (Life Technologies) and transferred to a $0.45-\mu \mathrm{m}$ nitrocellulose membrane (VWR). For detection, either fluorophore-coupled secondary antibodies or HRP-conjugated secondary antibodies and WesternBright Chemiluminescent Substrate (Biozym Scientific) or SuperSignal West Pico Chemiluminescent Substrate (Life Technologies) were used. Western blots were quantified by determining the background-subtracted densities of the protein of interest using ImageJ [59]. The signal from the AP (against GFP-tagged protein of interest) was normalized to the respective control samples expressing the empty vector or to the input.

\section{Antibodies}

The following antibodies were used: anti-GFP (B-2 clone; Santa Cruz; sc-9996), anti-MKRN1 (Bethyl Laboratories, A300-990A), anti-PABPC1/3 (Cell Signaling, 4992), anti-ZNF598 (N1 N3; GeneTex; GTX119245), anti-ELAVL1 (Santa Cruz, sc-5261), anti-LARP1 (Santa Cruz, sc-515873), anti-Ubiquitin (P4D1; Santa Cruz, sc8017), anti-Vinculin (Sigma Aldrich, V9264), antiaTubulin (Sigma Aldrich, T-5168), anti-Rabbit IgG (Cell Signaling; 7074), anti-Mouse IgG (Cell Signaling; 7076), IRDye ${ }^{\circ}$ 680RD Goat anti-Mouse IgG (P/N 925-68070), and IRDye $800 \mathrm{CW}$ Goat anti-Rabbit IgG (P/N 92532211) (both LI-COR Biosciences GmbH).

\section{Polysomal fractionation}

HEK293T cells were subjected to gradient centrifugation for polysomal fractionation. Briefly, $4 \times 10^{6}$ cells were seeded in $15-\mathrm{cm}$ cell culture dishes and incubated overnight at $37^{\circ} \mathrm{C}$ in a humidified atmosphere with $5 \% \mathrm{CO}_{2}$. To stall translation, $100 \mu \mathrm{g} / \mathrm{ml}$ cycloheximide (CHX) were added $10 \mathrm{~min}$ prior to harvest. Cells were washed with $\mathrm{PBS} / \mathrm{CHX}(100 \mu \mathrm{g} / \mathrm{ml})$, and lysed in $750 \mu \mathrm{l}$ polysome lysis buffer $(140 \mathrm{mM} \mathrm{KCl}, 20 \mathrm{mM}$ Tris- $\mathrm{HCl} \mathrm{pH} 8.0$, $5 \mathrm{mM} \mathrm{MgCl} 2,0.5 \%$ NP40, $0.5 \mathrm{mg} / \mathrm{ml}$ heparin, $1 \mathrm{mM}$ DTT, $100 \mathrm{U} / \mathrm{ml}$ RNasin [Promega, Mannheim, Germany], $100 \mu \mathrm{g} /$ $\mathrm{ml} \mathrm{CHX}$ ). The cell debris was pelleted by centrifugation ( 5 min, $4{ }^{\circ} \mathrm{C}, 13,000 \mathrm{rpm}$ ), and $600 \mu \mathrm{l}$ of the cleared cell lysates were layered onto $11 \mathrm{ml} 10-50 \%$ continuous sucrose gradients. The sucrose gradients were subjected to ultracentrifugation at $35,000 \mathrm{rpm}$ for $2 \mathrm{~h}$ at $4{ }^{\circ} \mathrm{C}$ without break using an SW40 Ti rotor (Beckman Coulter, Brea, USA). Afterwards, 1-ml fractions were collected using a Gradient Station (BioComp Instruments, Fredericton, Canada). UVabsorbance was measured at $254 \mathrm{~nm}$. Protein was precipitated by adding trichloro acetic acid (TCA) to each fraction (final $10 \%[\mathrm{v} / \mathrm{v}]$ ) and incubation overnight at $4{ }^{\circ} \mathrm{C}$. The samples were centrifuged for $20 \mathrm{~min}$ at $4{ }^{\circ} \mathrm{C}$ and $13,000 \mathrm{rpm}$ and the resulting pellets were washed twice with ice-cold acetone, dissolved in $100 \mu \mathrm{l} 2 \times$ SDS loading buffer $(62.5 \mathrm{mM}$ Tris- $\mathrm{HCl}$ pH 6.8, $2.5 \mathrm{mM}$ DTT, 10\% glycerol [v/v], 1\% SDS $[\mathrm{w} / \mathrm{v}], 0.001 \%$ bromophenol blue) and heated for $5 \mathrm{~min}$ at $95^{\circ} \mathrm{C}$.

\section{Recombinant protein expression}

$\mathrm{N}$-His ${ }_{6}$-tagged hsRPS10, hsPABPC1, and hsMKRN1 variants were expressed from pET53-DEST in E. coli Rosetta $^{\text {Tm }} 2$ (DE3) pLysS (Novagen). Cells were grown in LB-Luria at $37^{\circ} \mathrm{C}$ and $160 \mathrm{rpm}$. For all MKRN1 variants, $50 \mu \mathrm{M} \mathrm{ZnCl}{ }_{2}$ was added to the growth medium. For the expression of His-RPS10 and His-MKRN1 variants, cells were chilled on ice at $\mathrm{OD}_{600}$ of $0.6-0.8$ and expression was induced by addition of IPTG $(0.5 \mathrm{mM}$ final concentration). Cells were further incubated at $18^{\circ} \mathrm{C}$ and 160 rpm for $16-21 \mathrm{~h}$. Cells expressing His-PABPC1 were grown at $37^{\circ} \mathrm{C}$ and $160 \mathrm{rpm}$ for $4 \mathrm{~h}$.

Cells were harvested by centrifugation $(4000 \times g, 15$ min, $\left.4{ }^{\circ} \mathrm{C}\right)$ and lysed in $38 \mathrm{ml}$ of lysis buffer $(50 \mathrm{mM}$ Tris- $\mathrm{HCl} \mathrm{pH} 8.0,500 \mathrm{mM} \mathrm{NaCl}, 15 \mathrm{mM}$ imidazole, 1 $\mathrm{mM}$ DTT, $1 \mathrm{mM} \mathrm{MgCl}_{2}$, Benzonase 1:5000 [Sigma]) per liter of initial culture volume using a high pressure homogenizer (Constant Systems, TS-T240). Lysates were cleared by centrifugation $\left(45,000 \times g, 30 \mathrm{~min}, 4{ }^{\circ} \mathrm{C}\right)$. All proteins, except His-MKRN1-RING ${ }^{\text {mut }}$, were purified as follows: Proteins were passed over a HisTrap FF $5 \mathrm{ml}$ column (GE Healthcare). After extensive washing with wash buffer (50 mM Tris- $\mathrm{HCl} \mathrm{pH}$ 8.0, $500 \mathrm{mM} \mathrm{NaCl}, 15$ $\mathrm{mM}$ imidazole), His-tagged proteins were eluted using wash buffer containing $300 \mathrm{mM}$ imidazole. Elution fractions were pooled and diluted 1:10 in heparin-binding buffer $(17 \mathrm{mM} \mathrm{NaP}$ i pH 7.4, $50 \mathrm{mM} \mathrm{NaCl}, 5 \%$ glycerol, 1 mM DTT), or in the case of His-MKRN1-WT and HisMKRN1-PAM2 ${ }^{\text {mut }}$, in anion exchange binding buffer (20 mM Tris- $\mathrm{HCl}$ pH 8.0, $30 \mathrm{mM} \mathrm{NaCl}, 5 \%$ glycerol, 1 $\mathrm{mM}$ DTT). The diluted proteins were subsequently passed over a HiTrap Heparin HP $5 \mathrm{ml}$ column (GE Healthcare; His-RPS10 and His-PABPC1), or a HiTrap Q HP $5 \mathrm{ml}$ column (GE Healthcare; His-MKRN1 variants). After washing with the respective binding buffer, recombinant proteins were eluted by running a linear gradient of $0-1.5 \mathrm{M} \mathrm{NaCl}$ in binding buffer over 20 
column volumes. Fractions containing the respective recombinant protein were pooled and concentrated using Amicon ${ }^{\bullet}$ Ultra-15 spin concentrators (Merck Millipore). Concentrated protein pools were run on a Superdex 200 Increase 10/300 GL, or HiLoad 16/60 Superdex 200 prep grade column (GE Healthcare) in gel filtration buffer (40 mM Na-HEPES pH 7.4, $100 \mathrm{mM} \mathrm{NaCl}, 1 \mathrm{mM} \mathrm{MgCl}$, $1 \mathrm{mM}$ DTT, $10 \%$ glycerol). Peak fractions containing the recombinant protein were pooled, aliquoted, and snapfrozen in liquid nitrogen. Frozen aliquots were stored at $-80^{\circ} \mathrm{C}$. All purification steps were performed using a Biorad NGC Quest Plus FPLC system. As His-MKRN1RING $^{\text {mut }}$ was prone to precipitation, it was isolated by a rapid one-step pulldown using Ni-NTA agarose beads (binding buffer: $20 \mathrm{mM}$ Tris- $\mathrm{HCl} \mathrm{pH} 7.5,500 \mathrm{mM} \mathrm{NaCl}$, $30 \mathrm{mM}$ imidazole; elution buffer: $20 \mathrm{mM}$ Tris- $\mathrm{HCl} \mathrm{pH}$ 7.5, $500 \mathrm{mM} \mathrm{NaCl}, 1 \mathrm{mM} \mathrm{MgCl} 2,500 \mathrm{mM}$ imidazole). 1 mM DTT and $10 \%$ glycerol were added to the eluted His-MKRN1-RING ${ }^{\text {mut }}$, and it was snap-frozen in liquid nitrogen.

\section{In vitro ubiquitylation assays}

Autoubiquitylation assay: To assess the functionality and capability of autoubiquitylation of recombinant MKRN1, His-MKRN1-WT $(1 \mu \mathrm{M})$, His-MKRN1-PAM2 ${ }^{\text {mut }}(1.5 \mu \mathrm{M})$, or His-MKRN1-RING ${ }^{\text {mut }}(1.5 \mu \mathrm{M})$ were incubated with or without E2 enzyme UBC5a $(2 \mu \mathrm{M})$, with E1 enzyme UBA1 $(0.25 \mu \mathrm{M})$ (IMB Protein Production Core Facility), ATP $(30 \mu \mathrm{M})$, ubiquitin $(5 \mu \mathrm{M}$; Sigma), and DTT $(1 \mathrm{mM})$ in $1 \times$ MAB reaction buffer (10× MAB reaction buffer: $400 \mathrm{mM}$ HEPES pH 7.4, $500 \mathrm{mM} \mathrm{NaCl}, 80 \mathrm{mM} \mathrm{MgCl}, 1 \mathrm{mM}$ DTT) for $1 \mathrm{~h}$ at $37^{\circ} \mathrm{C}$. As a control, the E2 enzyme Ubc5a was incubated with all reaction components without any E3 enzyme. The reaction was stopped by adding LDS sample buffer and boiling.

Ubiquitylation of target proteins: To assess whether recombinant MKRN1 is capable of ubiquitylating RPS10 and PABPC1, His-MKRN1-WT $(1 \mu \mathrm{M})$ was incubated with His-PABPC1 $(0.75 \mu \mathrm{M})$ or His-RPS10 $(0.75 \mu \mathrm{M})$, the E2 enzyme UBC5a $(2 \mu \mathrm{M})$, the E1 enzyme UBA1 $(0.25 \mu \mathrm{M})$, ATP $(30 \mu \mathrm{M})$, ubiquitin $(5 \mu \mathrm{M})$, and DTT (1 $\mathrm{mM}$ ) in $1 \times \mathrm{MAB}$ reaction for $1 \mathrm{~h}$ at $37^{\circ} \mathrm{C}$. As a control, His-PABPC1 or His-RPS10 were incubated with all reaction components without His-MKRN1-WT. The reaction was stopped by adding LDS sample buffer and boiling.

\section{RNA isolation, CDNA synthesis, and qPCR}

Cells were washed twice in ice-cold PBS and harvested. RNA was isolated using the RNeasy Plus Mini Kit (Qiagen) according to the manufacturer's recommendations. Five hundred nanograms of total RNA was transcribed into cDNA using random hexamer primers (Thermo Scientific) and the RevertAid Reverse Transcriptase (Thermo Scientific) according to the manufacturer's recommendations.
qPCR was performed using the Luminaris HiGreen qPCR Master Mix, low ROX (Thermo Scientific) according to the manufacturer's recommendations with $10 \mu \mathrm{M}$ forward and reverse primers (Additional file 1: Table S5).

\section{iCLIP experiments and data processing}

iCLIP libraries were prepared as described previously [60, 61]. HEK293T cells ectopically expressing either GFP alone (empty vector) or N-terminally GFP-tagged MKRN1 wild type (GFP-MKRN1 $1^{\text {wt }}$ ), GFP-MKRN1 $1^{\text {PAM2mut, }}$ or GFP-MKRN1 ${ }^{\text {RINGmut }}$ were used. For crosslinking, confluent cells were irradiated once with $150 \mathrm{~mJ} / \mathrm{cm}^{2}$ at $254 \mathrm{~nm}$ in a Stratalinker 2400 or treated with 4-thiouridine $(100 \mu \mathrm{M}$ for $16 \mathrm{~h}$ ) and irradiated with $3 \times 300 \mathrm{~mJ} / \mathrm{cm}^{2}$ in a Stratalinker 2400 with $365 \mathrm{~nm}$ bulbs. For IP, $10.5 \mu \mathrm{g}$ anti-GFP antibody (goat, Protein Unit, MPI-CBG, Dresden) was used per sample. The libraries were sequenced as 50-nt single-end reads on an Illumina MiSeq platform (Additional file 1: Table S2).

Basic sequencing quality checks were applied to all reads using FastQC (version 0.11.5) (https://www.bioinformatics. babraham.ac.uk/projects/fastqc/). Afterwards, reads were filtered based on sequencing qualities (Phred score) of the barcode region. Only reads with at most one position with a sequencing quality $<20$ in the experimental barcode (positions 4 to 7) and without any position with a sequencing quality $<17$ in the random barcode (positions 1-3 and 8-9) were kept for further analysis. Remaining reads were demultiplexed based on the experimental barcode on positions 4 to 7 using Flexbar (version 3.0.0) [62] without allowing mismatches.

All following steps of the analysis were performed on all individual samples after de-multiplexing. Remaining adapter sequences were trimmed from the right end of the reads using Flexbar (version 3.0.0) allowing up to one mismatch in $10 \mathrm{nt}$, requiring a minimal overlap of 1 nt of read and adapter. After trimming off the adapter, the barcode is trimmed off of the left end of the reads (first $9 \mathrm{nt}$ ) and added to the header of the read, such that the information is kept available for downstream analysis. Reads shorter than $15 \mathrm{nt}$ were removed from further analysis.

Trimmed and filtered reads were mapped to the human genome (assembly version GRCh38) and its annotation based on GENCODE release 25 [63] using STAR (version 2.5.4b) [64]. When running STAR, up to two mismatches were allowed, soft-clipping was prohibited at the $5^{\prime}$ ends of reads, and only uniquely mapping reads were kept for further analysis.

Following mapping, duplicate reads were marked using the dedup function of bamUtil (version 1.0.13), which defines duplicates as reads whose $5^{\prime}$ ends map to the same position in the genome (https://github.com/statgen/ bamUtil). Subsequently, marked duplicates with identical 
random barcodes were removed since they are considered technical duplicates, while biological duplicates showing unequal random barcodes were kept.

Resulting bam files were sorted and indexed using SAMtools (version 1.5) [65]. Based on the bam files, bedgraph files were created using bamToBed of the BEDTools suite (version 2.25.0) [66], considering only the position upstream of the $5^{\prime}$ mapping position of the read, since this nucleotide is considered as the crosslinked nucleotide. Bedgraph files were then transformed to bigWig file format using bedGraphToBigWig of the UCSC tool suite [67].

\section{Identification and characterization of MKRN1 binding sites}

Peak calling was performed on merged iCLIP coverage tracks (crosslink events per nucleotide) from the three replicates based on GENCODE annotation (release 27, GRCh38) using ASPeak (version 2.0; default setting plus -nornaseq to estimate parameters $p$ and $r$ for the negative binomial distributions in a 500-nt window around each peak) [68]. The initially predicted peaks were resized to uniform 9-nt windows around their weighted centered as defined by ASPeak. To avoid artifacts, we removed sparsely covered peaks that harbor crosslink events on less than three nucleotides within the 9-nt region window. We iteratively merged all remaining windows if overlapping by at least $1 \mathrm{nt}$, by defining the position with the cumulative half maximum count of crosslink events as new window center. We then excluded all windows overlapping with none or multiple protein-coding genes (GENCODE annotations support level $\geq 2$ and transcript support level $\geq 3$ ) and assigned each binding site to a distinct genomic region ( 3 ' UTR, 5' UTR, CDS, intron). Consistent with the mostly cytoplasmic localization of MKRN1 [18, 19, 21], less than 6\% of the binding sites were predicted within introns, which were excluded from further analysis. Finally, we kept only reproducible binding sites with at least three crosslink events in all three replicates. This procedure yielded a total of 7331 MKRN1 binding sites in 2163 genes (Additional file 1: Figure S4A).

In order to estimate binding site strength and to facilitate comparisons between binding sites (Fig. 2c, e, Additional file 1: Figure S4E-G and S5A,C,D), we corrected for transcript abundance by representing the crosslink events within a binding site as a "signal-over-background" ratio (SOB). The respective background was calculated as the sum of crosslink events outside of binding sites (plus $5 \mathrm{nt}$ to either side) by the merged length of all exons. 3' UTR lengths were restricted to $10 \mathrm{nt}$ past the last MKRN1 binding site or $500 \mathrm{nt}$ if no binding site was present. SOB calculations were performed separately for each replicate and then averaged. No SOB value was assigned for genes with a background of $<10$ crosslink events, resulting in $\mathrm{SOB}$ values for $97 \%$ of all binding sites.

In order to assess the local RNA sequence context of MKRN1 binding sites (Fig. 2c and Additional file 1: Figure S5A), enriched 4-mers were counted inside the 9nt binding sites as well as within 40-nt before and after. To estimate an empirical background distribution, 1000 9-nt windows were randomly picked in 3' UTRs and 4mer frequencies were counted in the same windows. This process was repeated 100 times, and the resulting mean and standard deviation were used to calculate the $z$-score for each 4-mer.

In order to define the A-rich regions downstream of MKRN1 binding sites in 3' UTRs (A-rich stretches), we used a maximization approach in a 55nt search space starting from the binding site center. Within this space, we calculated the percentage of A nucleotides (A-content) for windows of increasing size $(8-30 \mathrm{nt})$ and selected the stretch with highest value for each window size. In case of ties, the window closer to the binding site was preferred, resulting in a set of 23 candidate A-rich stretches with the maximal A-content for each length. Next, we computed the longest continuous A-run (LCA) and a weighted Acontent (multiplying the A-content with the number of A nucleotides) for each candidate A-rich stretch. Candidate A-rich stretches with an A-content $<70 \%$, a weighted A-content $<11$, and an $\mathrm{LCA}<4$ were excluded. The final A-rich stretch for each binding site was then selected in a hierarchical manner, preferring LCA over weighted A-content. Lastly, overlapping Arich stretches of neighboring binding sites were merged by selecting the highest scoring Arich stretch, based on LCA and weighted A-content. In total, this procedure identified 1412 nonoverlapping A-rich stretches, associated with 1848 binding sites.

In order to estimate the extent of MKRN1 binding to poly(A) tails (Fig. 3a), we evaluated the percentage of adenosine within the iCLIP reads that could not be mapped to the human genome without soft-clipping (see above). iCLIP data for heterogeneous nuclear ribonucleoprotein $\mathrm{H}$ (HNRNPH) served as control [33]. To further support MKRN1 binding upstream of poly(A) tails, we counted the number of continuous A's at the 3' ends of all unmapped reads (Fig. 3a, inset).

Annotated transcript $3^{\prime}$ ends (i.e., polyadenylation sites) were taken from GENCODE (all annotated protein-coding transcripts with support level $\leq 2$ and transcript support level $\leq 3$; release 28, GRCh38.p12; https://www.gencodegenes.org/). For comparison, we included seven publicly available eCLIP datasets (ENCODE project) [34] from human $\mathrm{K} 562$ cells for the RBPs PABPC4 (ENCODE accession number 
ENCFF440SQF), UPF1 (ENCFF466HWF), PUM1 (ENCFF019LLG), HNRNPK (ENCFF924WZQ), QKI (ENCFF120WPV), CPSF6 (ENCFF420PXR), and TIAL1 (ENCFF430UQQ). For each RBP, we used the mate \#2 fastq file from the replicate with more reads, which was processed as described above. For Fig. 3b, all crosslink events within a 2-kb window around the polyadenylation sites for $3^{\prime}$ UTR longer than $1 \mathrm{~kb}$ were counted. For each RBP, only 3' UTRs with at least 10 crosslink events in $2-\mathrm{kb}$ window were taken. The resulting crosslink profiles were normalized to the total number of considered regions for each RBP and smoothened with a running 50-nt window.

\section{RNA pulldown experiments}

The RNA pulldown experiments were performed with 42nt RNA oligonucleotides, containing 22 nt of the SRSF4 3' UTR (Fig. 3c) until the cleavage site (chr1:29,147,886-29, $147,907)$, including the proximal polyadenylation signal of SRSF4. This invariant part was followed, either by $20 \mathrm{~A}$ $\left(\mathrm{A}_{20}\right.$ RNA) or by a control sequence of $20 \mathrm{~V}$ (Control RNA). The RNA oligonucleotides were ordered from IDT. Fifty-picomole RNA oligonucleotides per reaction were biotinylated using the Pierce RNA 3' End Biotinylation Kit (Thermo Fisher Scientific) according to the manufacturer's recommendations. The biotinylated oligonucleotides were bound to High Capacity NeutrAvidin agarose beads (Thermo Fisher Scientific). NeutrAvidin agarose beads that were not coupled to biotinylated oligonucleotides were used as controls. After washing, one third of RNA-bound beads was incubated with $2 \mu \mathrm{g}$ recombinant His-MKRN1-WT and/or recombinant His-PABPC1, respectively, for $1 \mathrm{~h}$ at $4{ }^{\circ} \mathrm{C}$ in binding buffer $(50 \mathrm{mM}$ Tris$\mathrm{HCl} \mathrm{pH} \mathrm{7.4,} 500 \mathrm{mM} \mathrm{KCl,} 12.5 \mathrm{mM} \mathrm{MgCl}_{2}, 1 \%$ Triton X100). After three washes in washing buffer $(200 \mathrm{mM} \mathrm{NaCl}$, $10 \mathrm{mM} \mathrm{MgCl}_{2}, 50 \mathrm{mM}$ HEPES pH 7.4, 0.5\% Igepal CA630, $1 \%$ Triton X-100), proteins were eluted from the beads by boiling in NuPAGE LDS sample buffer (Thermo Fisher Scientific) for $10 \mathrm{~min}$ at $70^{\circ} \mathrm{C}$. Proteins were analyzed by SDS-PAGE and Coomassie staining.

\section{Evolutionary characterization of Makorin protein family}

Four different ortholog searches were performed using HaMStR-OneSeq [69] against the Quest for Orthologs Consortium protein set, containing 78 species (release 2017_04) [70]. For each run, a different seed protein was chosen: human MKRN1-3 (UniProt identifiers Q9UHC7, Q9H000, and Q13064) and MKRN4 from zebrafish (A9C4A6). In order to identify proteins with a similar domain architecture, we calculated a unidirectional feature architecture similarity (FAS) score which compares the domain architecture of the seed protein and the predicted ortholog [71]. Predicted orthologues with FAS $<0.7$ were removed after initial assessment. Finally, all vertebrate species and selected invertebrate species were used for reconstruction of a maximum likelihood (ML) tree. For this, protein sequences were aligned using MAFFT v7.294b L-INS-i [72], and ML trees with 100 bootstrap replicates were calculated using RAxML version 8.1.9 [73]. Settings for a rapid bootstrap analysis and searching for the best scoring ML tree in one program run (- $\mathrm{f} a)$ and an automatic selection of the best fitting amino acid substitution model (-m PROTGAMMAAUTO) were chosen. Reconstructed trees were visualized using FigTree v1.4.2 (http://tree.bio.ed.ac.uk/ software/figtree/).

The phylogenetic tree and FASTA sequences from the ortholog dataset were loaded into DoMosaics [74], and Pfam domains were annotated with HMMER (http://hmmer.org/, default parameters). We additionally searched for the PAM2 motif that interacts with the MLLE domain of PABP proteins $[75,76]$.

Since the PAM2 motif in all Makorin proteins differs from the described consensus motif [22], a custom Hidden Markov Model was trained on PAM2 motifs from selected Makorin orthologs and used for a HMMER scan of the orthologs (no E-value cutoff). The same procedure was repeated for the recently reported PAM2-like motif (PAM2L) [25].

\section{Immunofluorescence microscopy}

HEK293T cells were seeded on microscopy cover slips and transfected with GFP-tagged MKRN1 $1^{\text {wt }}$ or MKRN1 $1^{\text {PAM2mut }}$. Cells were washed with ice-cold PBS and fixed with $4 \%$ paraformaldehyde (Affymetrix) for $20 \mathrm{~min}$. Cells were stained with DAPI (Sigma) and rinsed in PBS, wash buffer (10 mM Tris- $\mathrm{HCl} \mathrm{pH} \mathrm{7.5),} \mathrm{and} \mathrm{water.} \mathrm{The} \mathrm{samples} \mathrm{were}$ mounted with ProLong Diamond Antifade Mountant (Life Technologies). For analysis, a Leica SP5 II confocal system (Leica Microsystems) with a $\times 63$ oil immersion NA1.4 objective lens was used, and two pictures were taken per frame. Images were processed in Fiji [77].

\section{Dual fluorescence translation stall assay via flow cytometry}

Knockdowns were performed for $24 \mathrm{~h}$, before the dual fluorescence reporter plasmids were ectopically expressed for $48 \mathrm{~h}$. Cells were washed in PBS and trypsinized. After sedimentation, cells were resuspended in DPBS supplemented with 2 $\mathrm{mM}$ EDTA. Cellular GFP and RFP fluorescence was measured using flow cytometry on a LSRFortessa SORP (BD Biosciences). Data analysis was done using Flowjo (v10) (FlowJo, LLC). For statistical testing, paired two-tailed Student's $t$ tests with Benjamini-Hochberg correction were performed on $n \geq 6$ replicates.

\section{Complementation approach}

For complementation experiments, cell lines stably expressing siRNA2-insensitive MKRN1 variants were 
used. To this end, HEK293T cells were transfected with plasmids coding for siRNA2-insensitive $M K R N 1^{w t}$, $M K R N 1^{\text {PAM2mut }}$, or $M K R N 1^{R I N G m u t}$ or an empty vector (EV) control using PEI as described above. Forty-eight hours after transfection, puromycin selection was started $(1.5 \mu \mathrm{g} / \mathrm{ml}$ puromycin). Upon single-cell dilutions, cell lines that stably expressed $M K R N 1^{w t}$, mutants or EV were kept in DMEM containing $1.5 \mu \mathrm{g} / \mathrm{ml}$ puromycin. In the dual fluorescence translation stall assay via flow cytometry, cells were seeded in DMEM without puromycin for $24 \mathrm{~h}$. Then, knockdowns were performed for $24 \mathrm{~h}$, before the dual fluorescence reporter plasmids were ectopically expressed for $48 \mathrm{~h}$. Cells were prepared and analyzed as described above.

\section{CRISPR/Cas9 knockout}

The pSpCas9(BB)-2A-Puro (PX459) V2.0 backbone (Addgene \#62988) was digested with BbsI, dephosphorylated, and gel-purified using the Qiagen QIAquick Gel Extraction Kit according to the manufacturer's recommendations [78, 79]. MKRN1 guide RNAs (see Additional file 1: Table S5) were designed using the ChopChop Website (http://chopchop.cbu.uib.no) [80, 81]. Phosphorylated oligonucleotides were annealed. Using T4 DNA ligase at $16^{\circ} \mathrm{C}$ overnight, the oligonucleotides were ligated into the digested vector [78, 79]. The CRISPR-SpCas9 plasmid containing guide RNA targeting MKRN1 was transfected into HEK293T cells. After $48 \mathrm{~h}$, CRISPR-SpCas9-positive cells were selected using puromycin $(1.5 \mu \mathrm{g} / \mathrm{ml})$ for 5 days. MKRN1 KO cells were subsequently cultured in DMEM with $10 \%$ fetal bovine serum, $1 \%$ penicillin/streptomycin, and 1\% L-glutamine. Upon single-cell dilutions, MKRN1 protein levels of these cells were assessed by Western Blot, and MKRN1 mRNA levels were analyzed by qPCR.

\section{Ubiquitin remnant profiling}

Di-glycine remnant profiling was performed as described before [82, 83]. In four different experiments, isotope labels were assigned as follows: experiment 1, MKRN1 KD1 (siRNA1), MKRN1 KD2 (siRNA2), and control siRNA with light, medium, and heavy SILAC labels, respectively; experiment 2, MKRN1 KD2 (siRNA2) and control siRNA with heavy and light SILAC labels, respectively; experiment 3, MKRN1 KD2 (siRNA2) and control siRNA with heavy and light SILAC labels, respectively; experiment 3, MKRN1 KD2 (siRNA2) and control siRNA with light and heavy SILAC labels, respectively. Cells were treated with the proteasome inhibitors bortezomib $(1 \mu \mathrm{M}, 8 \mathrm{~h}$, replicate 1 ; Santa Cruz Biotechnology) or MG132 (10 $\mu \mathrm{M}, 2 \mathrm{~h}$, replicates 2, 3, 4; Sigma). Proteins were precipitated in acetone. Proteins were digested with endoproteinase Lys-C (Wako Chemicals) and sequencing-grade modified trypsin (Sigma). To purify the peptides, reversed-phase Sep-Pak C18 cartridges (Waters) were used. Modified peptides were enriched using di-glycine-lysine antibody resin (Cell Signaling Technology). The enriched peptides were eluted with $0.15 \%$ trifluoroacetic acid in water, then fractionated using micro-column-based strong-cation exchange chromatography (SCX) [84] before being desalted on reversed-phase C18 StageTips [49]. Samples were analyzed by quantitative mass spectrometry and MaxQuant as described above. To identify significantly regulated ubiquitylation sites, the limma algorithm was applied [56]. A $P$ value $<0.1$ after multiple testing correction was used as a cutoff to determine up- and downregulated ubiquitylation sites. Volcano and dot plots were created in R (version 3.4.3).

\section{Functional interaction network of MKRN1 ubiquitylation target proteins}

The functional protein interaction network analysis was performed by integrating interaction data from the STRING database $($ score $>0.4)$, the BioGrid database, and our own findings $[85,86]$. Cytoscape (version 3.6.1) was used to visualize the protein interaction network [87].

\section{Supplementary information}

Supplementary information accompanies this paper at https://doi.org/10. 1186/s13059-019-1814-0.

\begin{abstract}
Additional file 1: Figure S1. Maximum likelihood tree of Makorin orthologs with their protein domain architecture. Figure S2. MKRN1 interacts with translational regulators and other RBPS. Figure S3. GFPMKRN1 ${ }^{\text {RINGmut }}$ interacts with PABPC1/4 and RPS10. Figure S4. Signalover-background transformation allows to estimate MKRN1 binding site strength. Figure S5. MKRN1 binds upstream of A-rich stretches. Figure S6. Interaction with PABPC1 is required for MKRN1 RNA binding. Figure S7. MKRN1 is required to stall ribosomes at $K(A A A)_{20}$ in reporter assays. Figure S8. Cross-regulation of MKRN1 and ZNF598. Figure S9. Proteome analysis upon MKRN1 KD and GO term analysis of MKRN1 ubiquitylation targets. Table S2. Summary of MKRN1 iCLIP experiments. Table S5 Oligonucleotides used in this study. Table S6 siRNAs used in this study. (PDF $2450 \mathrm{~kb}$ )
\end{abstract}

Additional file 2: Table S1. with MaxQuant analysis of MS data from the SILAC-AP for GFP-MKRN1 $1^{\text {wt }}$, GFP-MKRN1 $1^{\text {PAM } 2 \text { mut }}$, and GFP-MKRN $1^{\text {RINGmut }}$ (XLSX 1409 kb)

Additional file 3: Figures S10-S12. with images of full membranes and different exposure times for Western blots and other analyses as specified. (PDF $2348 \mathrm{~kb}$ )

Additional file 4: Table S3. with MaxQuant analysis of MS data from the ubiquitin remnant profiling from MKRN1 KD2 in HEK293T cells. (XLSX $7940 \mathrm{~kb})$

Additional file 5: Table S4. with MaxQuant analysis of MS data from the proteome analysis from MKRN1 KD2 in HEK293T cells. (XLSX 2270 kb)

Additional file 6: Review history. (DOCX $34 \mathrm{~kb}$ )

\section{Acknowledgements}

We would like to thank all members of the Zarnack, König, and Beli labs, as well as René Ketting, Nadine Wittkopp, and Miguel Almeida for fruitful discussions. The authors gratefully thank the Ramanujan S. Hegde for providing the dual fluorescence reporter plasmids. The authors thank Christian Renz, Núria Casas Vila, Vivien Schoonenberg, Michael Musheev, 
Nadezda Podvalnaya, and Nicola Böffinger for experimental help. We thank Elmar Wahle, Uwe Kühn, and Filip Pekovic for help with PABPC1 expression. We thank Anja Freiwald for assistance with mass spectrometry analysis and Stefan Simm for assistance with evaluating PAM2 mutations. The support of the IMB Core Facilities Bioinformatics, Flow Cytometry, Genomics, and the use of its Illumina MiSeq, Protein Production, Microscopy, as well as the DFG-funded Mass Spectrometer Q Exactive Plus (INST 247/766-1 FUGG), are gratefully acknowledged. Animal shapes in Additional file 1: Figure S1A were obtained from PhyloPic and are used under the Creative Common Attribution-NonCommercial-ShareAlike 3.0 Unported license. We acknowledge the ENCODE Consortium and the Yeo lab for the eCLIP data analyzed in this study.

\section{Review history}

The review history is available as Additional file 6.

\section{Authors' contributions}

AH performed iCLIP experiments, flow cytometry measurements of dual fluorescence reporters, QPCR experiments, and most proteomics experiments. MB performed most bioinformatics analyses of MKRN1 iCLIP data. CR analyzed the binding of MKRN1 and other RBPs at polyadenylation sites and poly(A) tails. AB and SB performed initial iCLIP data processing and analysis. $A H$ and $A B$ analyzed the proteomics data. JBH and $A V$ contributed to replicate ubiquitin remnant profiling experiments and AP-Western blot experiments, respectively. AV performed in vitro ubiquitylation experiments. $\mathrm{HH}$ performed replicate iCLIP, QPCR, and replicate AP-Western blot experiments. CR and IE contributed to the evolutionary characterization of Makorin proteins. AS and TS performed polysome fractionation experiments. MM purified recombinant proteins. $A D$ and J-YR performed complementary studies in D. melanogaster. SE and KZ supervised the bioinformatics analyses. JK and PB conceived the project with $\mathrm{KZ}$ and supervised the experimental work. $\mathrm{AH}$, JK, $\mathrm{KZ}$, and PB wrote the manuscript with help and comments from all coauthors. All authors read and approved the final manuscript.

\section{Funding}

The project was funded by the German Research Foundation (DFG) as part of SPP1925 to J.-Y.R. (RO 4681/4-1) and J.K. (KO 4566/3-1). P.B. is supported by the Emmy Noether Program (BE 5342/1-1) and the SFB1177 of the German Research Foundation. K.Z. was supported by the LOEWE program Ubiquitin Networks (Ub-Net) of the State of Hesse (Germany) and the SFB 902 of the German Research Foundation.

\section{Availability of data and materials}

Dataset supporting the conclusions of this article are available at the ProteomeXchange Consortium (http://proteomecentral.proteomexchange.org/ cgi/GetDataset?ID=PXD011772) [88] via the PRIDE partner repository with the identifier PXD011772 (https://www.ebi.ac.uk/pride/archive/projects/PXD011772) (proteomics) and the Gene Expression Omnibus under the accession number GSE122869 (https://www.ncbi.n/m.nih.gov/geo/query/acc.cgi?acc=GSE122869) (iCLIP) [89].

eCLIP datasets analyzed during the current study were retrieved from the ENCODE data portal (https://www.encodeproject.org/) [34] via accession numbers ENCFF440SQF (PABPC4), ENCFF466HWF(UPF1), ENCFF019LLG (PUM1), ENCFF924WZQ (HNRNPK), ENCFF120WPV (QKI), ENCFF420PXR (CPSF6), and ENCFF430UQQ (TIAL1).

\section{Ethics approval and consent to participate}

The cell lines were authenticated by immunology, PCR, and cytogenetics by DSMZ (https://www.dsmz.de/collection/catalogue/details/culture/ACC-635).

\section{Competing interests}

The authors declare that they have no competing interests.

\section{Author details}

${ }^{1}$ Institute of Molecular Biology (IMB), Ackermannweg 4, 55128 Mainz, Germany. ${ }^{2}$ Buchmann Institute for Molecular Life Sciences (BMLS), Goethe University, Max-von-Laue-Str. 15, 60438 Frankfurt am Main, Germany. ${ }^{3}$ Faculty of Medicine, Institute of Biochemistry I, Goethe University Frankfurt, Theodor-Stern-Kai 7, 60590 Frankfurt am Main, Germany. ${ }^{4}$ Department for Applied Bioinformatics, Institute of Cell Biology and Neuroscience, Goethe University Frankfurt, Max-von-Laue-Str. 13, 60438 Frankfurt am Main,
Germany. ${ }^{5}$ Senckenberg Biodiversity and Climate Research Centre (BiK-F), Georg-Voigt-Straße 14-16, 60325 Frankfurt am Main, Germany. ${ }^{6}$ Center for Integrative Genomics, Faculty of Biology and Medicine, University of Lausanne, Génopode Building, CH-1015 Lausanne, Switzerland.

\section{Received: 26 January 2019 Accepted: 4 September 2019}

Published online: 22 October 2019

\section{References}

1. Chu J, Hong NA, Masuda CA, Jenkins BV, Nelms KA, Goodnow CC, et al. A mouse forward genetics screen identifies LISTERIN as an E3 ubiquitin ligase involved in neurodegeneration. Proc Natl Acad Sci U S A. 2009;106(7):2097-103.

2. Kaida D, Berg MG, Younis I, Kasim M, Singh LN, Wan L, et al. U1 snRNP protects pre-mRNAs from premature cleavage and polyadenylation. Nature. 2010;468(7324):664-8.

3. Bengtson $\mathrm{MH}$, Joazeiro CA. Role of a ribosome-associated E3 ubiquitin ligase in protein quality control. Nature. 2010;467(7314):470-3.

4. Joazeiro CAP. Ribosomal stalling during translation: providing substrates for ribosome-associated protein quality control. Annu Rev Cell Dev Biol. 2017;33:343-68.

5. Brandman O, Hegde RS. Ribosome-associated protein quality control. Nat Struct Mol Biol. 2016;23(1):7-15

6. Brandman O, Stewart-Ornstein J, Wong D, Larson A, Williams CC, Li GW, et al. A ribosome-bound quality control complex triggers degradation of nascent peptides and signals translation stress. Cell. 2012;151(5):1042-54.

7. Verma R, Oania RS, Kolawa NJ, Deshaies RJ. Cdc48/p97 promotes degradation of aberrant nascent polypeptides bound to the ribosome. eLife. 2013;2:e00308.

8. Juszkiewicz S, Hegde RS. Initiation of quality control during poly(A) translation requires site-specific ribosome ubiquitination. Mol Cell. 2017:65(4):743-50 e4.

9. Garzia A, Jafarnejad SM, Meyer C, Chapat C, Gogakos T, Morozov P, et al. The E3 ubiquitin ligase and RNA-binding protein ZNF598 orchestrates ribosome quality control of premature polyadenylated mRNAs. Nat Commun. 2017:8:16056.

10. Sundaramoorthy E, Leonard M, Mak R, Liao J, Fulzele A, Bennett EJ. ZNF598 and RACK1 regulate mammalian ribosome-associated quality control function by mediating regulatory 405 ribosomal ubiquitylation. Mol Cell. 2017;65(4):751-60 e4.

11. Juszkiewicz S, Chandrasekaran V, Lin Z, Kraatz S, Ramakrishnan V, Hegde RS ZNF598 is a quality control sensor of collided ribosomes. Mol Cell. 2018; 72(3):469-81 e7.

12. Gray TA, Hernandez L, Carey AH, Schaldach MA, Smithwick MJ, Rus K, et al. The ancient source of a distinct gene family encoding proteins featuring RING and C (3) $\mathrm{H}$ zinc-finger motifs with abundant expression in developing brain and nervous system. Genomics. 2000;66(1):76-86.

13. Böhne A, Darras A, D'Cotta H, Baroiller JF, Galiana-Arnoux D, Volff JN. The vertebrate makorin ubiquitin ligase gene family has been shaped by largescale duplication and retroposition from an ancestral gonad-specific, maternal-effect gene. BMC Genomics. 2010;11:721.

14. Kim JH, Park SM, Kang MR, Oh SY, Lee TH, Muller MT, et al. Ubiquitin ligase MKRN1 modulates telomere length homeostasis through a proteolysis of hTERT. Genes Dev. 2005;19(7):776-81.

15. Salvatico J, Kim JH, Chung IK, Muller MT. Differentiation linked regulation of telomerase activity by Makorin-1. Mol Cell Biochem. 2010; 342(1-2):241-50.

16. Omwancha J, Zhou XF, Chen SY, Baslan T, Fisher CJ, Zheng Z, et al. Makorin RING finger protein 1 (MKRN1) has negative and positive effects on RNA polymerase II-dependent transcription. Endocrine. 2006; 29(2):363-73.

17. Lee EW, Lee MS, Camus S, Ghim J, Yang MR, Oh W, et al. Differential regulation of $\mathrm{p} 53$ and $\mathrm{p} 21$ by MKRN1 E3 ligase controls cell cycle arrest and apoptosis. EMBO J. 2009;28(14):2100-13.

18. Cassar PA, Carpenedo RL, Samavarchi-Tehrani P, Olsen JB, Park CJ, Chang WY, et al. Integrative genomics positions MKRN1 as a novel ribonucleoprotein within the embryonic stem cell gene regulatory network. EMBO Rep. 2015;16(10):1334-57.

19. Miroci H, Schob C, Kindler S, Olschlager-Schutt J, Fehr S, Jungenitz T, et al. Makorin ring zinc finger protein 1 (MKRN1), a novel poly(A)-binding proteininteracting protein, stimulates translation in nerve cells. J Biol Chem. 2012; 287(2):1322-34 
20. Simsek D, Tiu GC, Flynn RA, Byeon GW, Leppek K, Xu AF, et al. The mammalian ribo-interactome reveals ribosome functional diversity and heterogeneity. Cell. 2017;169(6):1051-65 e18.

21. Hildebrandt A, Alanis-Lobato G, Voigt A, Zarnack K, Andrade-Navarro MA, Beli $P$, et al. Interaction profiling of RNA-binding ubiquitin ligases reveals a link between posttranscriptional regulation and the ubiquitin system. Sci Rep. 2017;7(1):16582.

22. Albrecht M, Lengauer T. Survey on the PABC recognition motif PAM2 Biochem Biophys Res Commun. 2004;316(1):129-38.

23. Kozlov G, Menade M, Rosenauer A, Nguyen L, Gehring K. Molecular determinants of PAM2 recognition by the MLLE domain of poly(A)-binding protein. J Mol Biol. 2010;397(2):397-407.

24. Deo RC, Sonenberg N, Burley SK. X-ray structure of the human hyperplastic discs protein: an ortholog of the C-terminal domain of poly(A)-binding protein. Proc Natl Acad Sci U S A. 2001;98(8):4414-9.

25. Pohlmann T, Baumann S, Haag C, Albrecht M, Feldbrügge M. A FYVE zinc finger domain protein specifically links mRNA transport to endosome trafficking. eLife. 2015;4:e06041.

26. König J, Zarnack K, Rot G, Curk T, Kayikci M, Zupan B, et al. iCLIP reveals the function of hnRNP particles in splicing at individual nucleotide resolution. Nat Struct Mol Biol. 2010;17(7):909-15.

27. Hafner M, Landthaler M, Burger L, Khorshid M, Hausser J, Berninger P, et al. Transcriptome-wide identification of RNA-binding protein and microRNA target sites by PAR-CLIP. Cell. 2010;141(1):129-41.

28. Sutandy FXR, Ebersberger S, Huang L, Busch A, Bach M, Kang HS, et al. In vitro iCLIP-based modeling uncovers how the splicing factor U2AF2 relies on regulation by cofactors. Genome Res. 2018;28(5):699-713.

29. Webster MW, Chen YH, Stowell JAW, Alhusaini N, Sweet T, Graveley BR, et al. mRNA deadenylation is coupled to translation rates by the differential activities of Ccr4-Not nucleases. Mol Cell. 2018;70(6):1089100 e8

30. Bag J. Feedback inhibition of poly(A)-binding protein mRNA translation. A possible mechanism of translation arrest by stalled $40 \mathrm{~S}$ ribosomal subunits. J Biol Chem. 2001;276(50):47352-60.

31. Lyabin DN, Eliseeva IA, Skabkina OV, Ovchinnikov LP. Interplay between Ybox-binding protein 1 (YB-1) and poly(A) binding protein (PABP) in specific regulation of YB-1 mRNA translation. RNA Biol. 2011:8(5):883-92.

32. Kini HK, Silverman IM, Ji X, Gregory BD, Liebhaber SA. Cytoplasmic poly(A) binding protein-1 binds to genomically encoded sequences within mammalian mRNAs. RNA. 2016;22(1):61-74.

33. Braun S, Enculescu M, Setty ST, Cortés-López M, de Almeida BP, Sutandy $\mathrm{FXR}$, et al. Decoding a cancer-relevant splicing decision in the RON protooncogene using high-throughput mutagenesis. Nat Commun. 2018;9(1): 3315.

34. Encode Project Consortium. An integrated encyclopedia of DNA elements in the human genome. Nature. 2012;489(7414):57-74.

35. Wang Z, Kayikci M, Briese M, Zarnack K, Luscombe NM, Rot G, et al. iCLIP predicts the dual splicing effects of TIA-RNA interactions. PLoS Biol. 2010; 8(10):e1000530.

36. Hurt JA, Robertson AD, Burge CB. Global analyses of UPF1 binding and function reveal expanded scope of nonsense-mediated mRNA decay. Genome Res. 2013;23(10):1636-50.

37. Ray D, Kazan H, Cook KB, Weirauch MT, Najafabadi HS, Li X, et al. A compendium of RNA-binding motifs for decoding gene regulation. Nature. 2013;499(7457):172-7.

38. Martin G, Gruber AR, Keller W, Zavolan M. Genome-wide analysis of premRNA $3^{\prime}$ end processing reveals a decisive role of human cleavage factor in the regulation of 3' UTR length. Cell Rep. 2012;1(6):753-63.

39. Lin YJ, Huang LH, Huang CT. Enhancement of heterologous gene expression in Flammulina velutipes using polycistronic vectors containing a viral 2A cleavage sequence. PLoS One. 2013;8(3):e59099.

40. Simms CL, Yan LL, Zaher HS. Ribosome collision is critical for quality control during no-go decay. Mol Cell. 2017;68(2):361-73 e5.

41. Letzring DP, Wolf AS, Brule CE, Grayhack EJ. Translation of CGA codon repeats in yeast involves quality control components and ribosomal protein L1. RNA. 2013;19(9):1208-17.

42. Lu J, Deutsch C. Electrostatics in the ribosomal tunnel modulate chain elongation rates. J Mol Biol. 2008;384(1):73-86.

43. Arthur L, Pavlovic-Djuranovic S, Smith-Koutmou K, Green R, Szczesny P, Djuranovic S. Translational control by lysine-encoding A-rich sequences. Sci Adv. 2015;1(6):e1500154.
44. Winz ML, Peil L, Turowski TW, Rappsilber J, Tollervey D. Molecular interactions between Hel2 and RNA supporting ribosome-associated quality control. Nat Commun. 2019;10(1):563.

45. Choe YJ, Park SH, Hassemer T, Korner R, Vincenz-Donnelly L, Hayer-Hartl M, et al. Failure of RQC machinery causes protein aggregation and proteotoxic stress. Nature. 2016;531(7593):191-5.

46. Jamar NH, Kritsiligkou P, Grant CM. Loss of mRNA surveillance pathways results in widespread protein aggregation. Sci Rep. 2018;8(1):3894.

47. Collaboration O. The ORFeome Collaboration: a genome-scale human ORFclone resource. Nat Methods. 2016;13(3):191-2.

48. Nielsen ML, Vermeulen M, Bonaldi T, Cox J, Moroder L, Mann M. lodoacetamide-induced artifact mimics ubiquitination in mass spectrometry. Nat Methods. 2008:5(6):459-60.

49. Rappsilber J, Mann M, Ishihama Y. Protocol for micro-purification, enrichment, pre-fractionation and storage of peptides for proteomics using StageTips. Nat Protoc. 2007;2(8):1896-906.

50. Michalski A, Damoc E, Hauschild JP, Lange O, Wieghaus A, Makarov A, et al. Mass spectrometry-based proteomics using Q Exactive, a high-performance benchtop quadrupole Orbitrap mass spectrometer. Mol Cell Proteomics. 2011;10(9):M111 011015.

51. Olsen JV, Macek B, Lange O, Makarov A, Horning S, Mann M. Higherenergy C-trap dissociation for peptide modification analysis. Nat Methods. 2007:4(9):709-12.

52. Cox J, Matic I, Hilger M, Nagaraj N, Selbach M, Olsen JV, et al. A practical guide to the MaxQuant computational platform for SILAC-based quantitative proteomics. Nat Protoc. 2009;4(5):698-705.

53. Cox J, Neuhauser N, Michalski A, Scheltema RA, Olsen JV, Mann M. Andromeda: a peptide search engine integrated into the MaxQuant environment. J Proteome Res. 2011;10(4):1794-805

54. Cox J, Mann M. MaxQuant enables high peptide identification rates, individualized p.p.b.-range mass accuracies and proteome-wide protein quantification. Nat Biotechnol. 2008;26(12):1367-72.

55. Elias JE, Gygi SP. Target-decoy search strategy for increased confidence in large-scale protein identifications by mass spectrometry. Nat Methods. 2007;4(3):207-14.

56. Ritchie ME, Phipson B, Wu D, Hu Y, Law CW, Shi W, et al. limma powers differential expression analyses for RNA-sequencing and microarray studies. Nucleic Acids Res. 2015:43(7):e47.

57. Jiao X, Sherman BT, Huang da W, Stephens R, Baseler MW, Lane HC, et al. DAVID-WS: a stateful web service to facilitate gene/protein list analysis. Bioinformatics. 2012;28(13):1805-6.

58. Supek F, Bosnjak M, Skunca N, Smuc T. REVIGO summarizes and visualizes long lists of gene ontology terms. PLoS One. 2011;6(7):e21800.

59. Schindelin J, Rueden CT, Hiner MC, Eliceiri KW. The ImageJ ecosystem: an open platform for biomedical image analysis. Mol Reprod Dev. 2015; 82(7-8):518-29.

60. Sutandy FXR, Hildebrandt A, König J. Profiling the binding sites of RNAbinding proteins with nucleotide resolution using iCLIP. Methods Mol Biol. 2016:1358:175-95.

61. Huppertz I, Attig J, D'Ambrogio A, Easton LE, Sibley CR, Sugimoto Y, et al. iCLIP: protein-RNA interactions at nucleotide resolution. Methods. 2014;65(3):274-87.

62. Dodt M, Roehr JT, Ahmed R, Dieterich C. FLEXBAR-flexible barcode and adapter processing for next-generation sequencing platforms. Biology. 2012:1(3):895-905

63. Harrow J, Frankish A, Gonzalez JM, Tapanari E, Diekhans M, Kokocinski F, et al. GENCODE: the reference human genome annotation for The ENCODE Project. Genome Res. 2012;22(9):1760-74.

64. Dobin A, Davis CA, Schlesinger F, Drenkow J, Zaleski C, Jha S, et al. STAR: ultrafast universal RNA-seq aligner. Bioinformatics. 2013;29(1):15-21.

65. Li H, Handsaker B, Wysoker A, Fennell T, Ruan J, Homer N, et al. The sequence alignment/map format and SAMtools. Bioinformatics. 2009;25(16): 2078-9.

66. Quinlan AR, Hall IM. BEDTools: a flexible suite of utilities for comparing genomic features. Bioinformatics. 2010;26(6):841-2

67. Kent WJ, Zweig AS, Barber G, Hinrichs AS, Karolchik D. BigWig and BigBed: enabling browsing of large distributed datasets. Bioinformatics. 2010:26(17):2204-7.

68. Kucukural A, Ozadam H, Singh G, Moore MJ, Cenik C. ASPeak: an abundance sensitive peak detection algorithm for RIP-Seq Bioinformatics. 2013;29(19):2485-6. 
69. Ebersberger I, Simm S, Leisegang MS, Schmitzberger P, Mirus O, von Haeseler $\mathrm{A}$, et al. The evolution of the ribosome biogenesis pathway from a yeast perspective. Nucleic Acids Res. 2014;42(3):1509-23.

70. Sonnhammer EL, Gabaldon T, Sousa da Silva AW, Martin M, RobinsonRechavi M, Boeckmann B, et al. Big data and other challenges in the quest for orthologs. Bioinformatics. 2014;30(21):2993-8.

71. Koestler T, von Haeseler A, Ebersberger I. FACT: functional annotation transfer between proteins with similar feature architectures. BMC Bioinformatics. 2010;11:417.

72. Katoh K, Standley DM. MAFFT multiple sequence alignment software version 7: improvements in performance and usability. Mol Biol Evol. 2013;30(4):772-80

73. Stamatakis A. RAxML version 8: a tool for phylogenetic analysis and postanalysis of large phylogenies. Bioinformatics. 2014;30(9):1312-3.

74. Moore AD, Held A, Terrapon N, Weiner J, Bornberg-Bauer E. DoMosaics: software for domain arrangement visualization and domain-centric analysis of proteins. Bioinformatics 2014;30(2):282-83.

75. Kozlov G, Trempe JF, Khaleghpour K, Kahvejian A, Ekiel I, Gehring K. Structure and function of the C-terminal PABC domain of human poly(A)binding protein. Proc Natl Acad Sci U S A. 2001;98(8):4409-13.

76. Kozlov G, De Crescenzo G, Lim NS, Siddiqui N, Fantus D, Kahvejian A, et al. Structural basis of ligand recognition by PABC, a highly specific peptidebinding domain found in poly(A)-binding protein and a HECT ubiquitin ligase. EMBO J. 2004;23(2):272-81.

77. Schindelin J, Arganda-Carreras I, Frise E, Kaynig V, Longair M, Pietzsch T, et al. Fiji: an open-source platform for biological-image analysis. Nat Methods. 2012;9(7):676-82

78. Ran FA, Hsu PD, Wright J, Agarwala V, Scott DA, Zhang F. Genome engineering using the CRISPR-Cas9 system. Nat Protoc. 2013;8(11):2281-308.

79. Cong L, Ran FA, Cox D, Lin S, Barretto R, Habib N, et al. Multiplex genome engineering using CRISPR/Cas systems. Science. 2013;339(6121):819-23.

80. Labun K, Montague TG, Gagnon JA, Thyme SB, Valen E. CHOPCHOP v2: a web tool for the next generation of CRISPR genome engineering. Nucleic Acids Res. 2016;44(W1):W272-6.

81. Montaque TG, Cruz JM, Gagnon JA, Church GM, Valen E. CHOPCHOP: a CRISPR/Cas9 and TALEN web tool for genome editing. Nucleic Acids Res. 2014;42(Web Server issue):W401-7.

82. Heidelberger JB, Voigt A, Borisova ME, Petrosino G, Ruf S, Wagner SA, et al. Proteomic profiling of VCP substrates links VCP to K6-linked ubiquitylation and c-Myc function. EMBO Rep. 2018;19(4):e44754.

83. Wagner SA, Beli P, Weinert BT, Nielsen ML, Cox J, Mann M, et al. A proteome-wide, quantitative survey of in vivo ubiquitylation sites reveals widespread regulatory roles. Mol Cell Proteomics. 2011;10(10):M111 013284.

84. Weinert BT, Scholz C, Wagner SA, lesmantavicius V, Su D, Daniel JA, et al. Lysine succinylation is a frequently occurring modification in prokaryotes and eukaryotes and extensively overlaps with acetylation. Cell Rep. 2013;4(4):842-51

85. Franceschini A, Szklarczyk D, Frankild S, Kuhn M, Simonovic M, Roth A, et al. STRING V9.1: protein-protein interaction networks, with increased coverage and integration. Nucleic Acids Res. 2013:41(Database issue):D808-15.

86. Chatr-Aryamontri A, Oughtred R, Boucher L, Rust J, Chang C, Kolas NK, et al. The BioGRID interaction database: 2017 update. Nucleic Acids Res. 2017; 45(D1):D369-D79.

87. Saito R, Smoot ME, Ono K, Ruscheinski J, Wang PL, Lotia S, et al. A travel guide to Cytoscape plugins. Nat Methods. 2012;9(11):1069-76.

88. Hildebrandt A, Brüggemann M, Rücklé C, Boerner S, Heidelberger JB, Busch $A$, et al. The RNA-binding ubiquitin ligase MKRN1 functions in ribosomeassociated quality control of poly(A) translation. ProteomeXchange Consortium. 2019. http://proteomecentral.proteomexchange.org/cgi/ GetDataset?ID=PXD011772. Accessed 19 Aug 2019

89. Hildebrandt A, Brüggemann M, Rücklé C, Boerner S, Heidelberger JB, Busch $A$, et al. The RNA-binding ubiquitin ligase MKRN1 functions in ribosomeassociated quality control of poly(A) translation. Gene Expression Omnibus. 2019; https:/www.ncbi.nlm.nih.gov/geo/query/acc.cgi?acc=GSE122869. Accessed 19 Aug 2019

\section{Publisher's Note}

Springer Nature remains neutral with regard to jurisdictional claims in published maps and institutional affiliations.

Ready to submit your research? Choose BMC and benefit from:

- fast, convenient online submission

- thorough peer review by experienced researchers in your field

- rapid publication on acceptance

- support for research data, including large and complex data types

- gold Open Access which fosters wider collaboration and increased citations

- maximum visibility for your research: over $100 \mathrm{M}$ website views per year

At BMC, research is always in progress.

Learn more biomedcentral.com/submissions 